Mathematical Programming manuscript No.

(will be inserted by the editor)

Mihai Anitescu $\cdot$ Paul Tseng $\cdot$ Stephen J. Wright

\title{
Elastic-Mode Algorithms for Mathematical Programs with Equilibrium Constraints: Global Convergence and Stationarity Properties
}

February 7, 2006

\begin{abstract}
The elastic-mode formulation of the problem of minimizing a nonlinear function subject to equilibrium constraints has appealing local properties in that, for a finite value of the penalty parameter, local solutions satisfying first- and second-order necessary optimality conditions for the original problem are also first- and second-order points of the elastic-mode formulation. Here we study global convergence properties of methods based on this formulation, which involve generating an (exact or inexact) first- or second-order point of the formulation, for nondecreasing values of the penalty parameter. Under certain regularity conditions on the active constraints, we establish finite or asymptotic convergence to points having a certain stationarity property (such as strong stationarity, M-stationarity, or C-stationarity). Numerical experience with these approaches is discussed. In particular, our analysis and the numerical evidence show that exact complementarity can be achieved finitely even when the elastic-mode formulation is solved inexactly.
\end{abstract}

Key words. Nonlinear programming, equilibrium constraints, complementarity constraints, elastic-mode formulation, strong stationarity, C-stationarity, Mstationarity.

AMS subject classifications 49M30, 49M37, 65K05, 90C30, 90C33

\section{Introduction}

We consider a mathematical program with equilibrium constraints (MPEC), defined as follows:

$$
\begin{array}{rl}
\min _{x} & f(x) \quad \text { subject to } \\
& g(x) \geq 0, \quad h(x)=0, \\
& 0 \leq G^{T} x \perp H^{T} x \geq 0,
\end{array}
$$

where $f: \mathbb{R}^{n} \rightarrow \mathbb{R}, g: \mathbb{R}^{n} \rightarrow \mathbb{R}^{p}$, and $h: \mathbb{R}^{n} \rightarrow \mathbb{R}^{q}$ are all twice continuously differentiable functions (at least in a neighborhood of all points generated by our methods), and $G$ and $H$ are $n \times m$ column submatrices of the $n \times n$ identity matrix (with no columns in common). Hence, the constraints $G^{T} x \geq 0$ and $H^{T} x \geq 0$ represent nonnegativity bound constraints on certain components of

M. Anitescu: Mathematics and Computer Science Division, Argonne National Laboratory, Argonne, IL 60439, e-mail: anitescu@mcs.anl.gov

P. Tseng: Department of Mathematics, University of Washington, Seattle, WA 98195, e-mail: tseng@math. washington.edu

S. J. Wright: Computer Sciences Department, University of Wisconsin, 1210 West Dayton Street, Madison, WI 53706, e-mail: swright@cs.wisc.edu 
$x$, and the notation $G^{T} x \perp H^{T} x$ signifies that $\left(G^{T} x\right)^{T}\left(H^{T} x\right)=0$. This special form of the complementarity constraints does not sacrifice generality; it can always be attained by introducing artificial variables as needed. We use this form because some of our results require the nonnegativity constraints $G^{T} x \geq 0$ and $H^{T} x \geq 0$ to be satisfied exactly even when $x$ is only an inexact solution of the subproblem in question. Such conditions are readily satisfied by most interior-point and active-set methods.

MPEC has been well studied in recent years, with many solution methods proposed; see $[2,3,5,12,14-16,18,20,22]$ and references therein. Although an MPEC can be formulated as a nonlinear program by rewriting the complementarity constraint as an equality constraint $\left(G^{T} x\right)^{T}\left(H^{T} x\right)=0$ or as an inequality constraint $\left(G^{T} x\right)^{T}\left(H^{T} x\right) \leq 0$, the resulting nonlinear program is highly degenerate; that is, it does not satisfy the linear independence constraint qualification (LICQ) nor the Mangasarian-Fromovitz constraint qualification (MFCQ). Thus, in order to achieve global convergence, specialized methods have been proposed that exploit the special structure of the complementarity constraint. These methods generate a sequence of points in $\mathbb{R}^{n}$ whose accumulation points satisfy, under suitable assumptions, certain necessary optimality conditions for the MPEC (1). Different types of necessary optimality conditions have been developed, the strongest and most desirable of which is strong stationarity [22]; see Definition 1 below. Under MPEC-LICQ (see Definition 2), strong stationarity is equivalent to the notion of B-stationarity [6]. Two weaker conditions, M-stationarity and C-stationarity [17,22], will also be of interest (see Definition 3).

A regularization method of Scholtes [23] achieves M-stationarity under MPECLICQ and achieves strong stationarity under an additional upper-level strict complementarity (ULSC) condition. A relaxation method of Lin and Fukushima [14] and a penalty method of Hu and Ralph [9], penalizing the complementarity constraint, have similar global convergence properties. A smoothing method of Fukushima and Pang [6] achieves strong stationarity under MPEC-LICQ and an additional asymptotically weak nondegeneracy condition. All these methods are conceptual, in that they assume the generation of a sequence of points satisfying exactly certain second-order necessary optimality conditions. Only in the case of linear constraints has a practical method been developed (Fukushima and Tseng [7]). We are led to ask: Can global convergence (to C- or M- or strongly stationarity points) be achieved under weaker assumptions or for more practical methods?

In this paper, we study this question for a nonlinear programming formulation of (1) that uses an explicit penalization of the complementarity constraint, also known as the "elastic mode." For a given penalty parameter $c \geq 0$ and fixed upper bound $\bar{\zeta} \in[0, \infty)$, this formulation can be written as follows:

$$
\begin{aligned}
\mathrm{PF}(c): \min _{x, \zeta} & f(x)+c \zeta+c\left(G^{T} x\right)^{T}\left(H^{T} x\right) \quad \text { subject to } \\
& g(x) \geq-\zeta e_{p}, \quad \zeta e_{q} \geq h(x) \geq-\zeta e_{q}, \quad 0 \leq \zeta \leq \bar{\zeta}, \\
& G^{T} x \geq 0, \quad H^{T} x \geq 0,
\end{aligned}
$$

where $e_{l}$ is the vector $(1,1, \ldots, 1)^{T}$ with $l$ components. A similar formulation was studied by Anitescu $[1,2]$, while a variant with $\zeta$ fixed at zero was investigated 
by Ralph and Wright [20]. The penalty method in [9] is based on this variant. Our analysis may also be extended to this variant, as well as to a mixed variant whereby $\zeta$ is fixed at zero for a subset of the constraints (see Section 5). A nice feature of (2) is that, for $\bar{\zeta}$ sufficiently large, a feasible point of (2) is easily found, so that (2) is solvable by feasible as well as infeasible methods. We will see that there are appealing correspondences between points $x^{*}$ that satisfy first-order optimality conditions for $(1)$ and points $\left(x^{*}, 0\right)$ that satisfy first-order optimality conditions for (2) (see Theorem 2).

The algorithms we consider in this paper generate a sequence of (exact or inexact) first- or second-order points $\left(x^{k}, \zeta_{k}\right)$ of $\mathrm{PF}\left(c_{k}\right)$, where $\left\{c_{k}\right\}$ is a positive nondecreasing sequence. We study the stationarity properties of the accumulation points of $\left\{x^{k}\right\}$. The upper bound constraint $\zeta_{k} \leq \bar{\zeta}$ helps to ensure the existence and boundedness of $\left(x^{k}, \zeta_{k}\right)$.

Our analyses draw on global convergence analyses of Scholtes [23] and Anitescu [2]; the latter studied a variant of (1) known as parametric mixed-P variational inequalities. In Section 3, we study stationarity properties of termination points and accumulation points of $\left\{\left(x^{k}, \zeta_{k}\right)\right\}$. In Subsection $3.1,\left(x^{k}, \zeta_{k}\right)$ is an inexact first-order point of $\operatorname{PF}\left(c_{k}\right)$, and we show that each feasible accumulation point satisfying MPEC-LICQ is C-stationary for (1). In Subsection 3.2, $\left(x^{k}, \zeta_{k}\right)$ is an exact second-order point of $\operatorname{PF}\left(c_{k}\right)$, and we show (somewhat surprisingly) termination at a strongly stationary point for $c_{k}$ sufficiently large; otherwise accumulation points either are infeasible or fail to satisfy MPEC-LICQ. In Subsection 3.3, $\left(x^{k}, \zeta_{k}\right)$ is an inexact second-order point of $\mathrm{PF}\left(c_{k}\right)$, and we show that each feasible accumulation point satisfying MPEC-LICQ is either Mstationary or strongly stationary (depending on boundedness of $\left\{c_{k}\right\}$ ). Moreover, if exact complementarity holds between bound constraints and their multipliers, then $x^{k}$ satisfies exactly the complementarity condition $\left(G^{T} x^{k}\right)^{T}\left(H^{T} x^{k}\right)=0$ for all $c_{k}$ sufficiently large. In Subsection 3.4, we introduce a strengthened version of MPEC-LICQ and prove another result concerning exact satisfaction of the complementarity condition for sufficiently large $c_{k}$-even when the subproblems $\mathrm{PF}\left(c_{k}\right)$ are solved inexactly. In Subsection 3.5, we present a practical algorithm for generating $\left(x^{k}, \zeta_{k}\right)$ as an inexact second-order point of $\mathrm{PF}\left(c_{k}\right)$.

Section 4 discusses a "regularized" nonlinear programming formulation of (1) [23] and presents examples to illustrate and compare the behavior of methods based on elastic-mode and regularized formulations. Section 5 presents some numerical experience, corroborating the aforementioned result of exact complementarity under finite penalty.

In what follows, we use $\|\cdot\|$ to denote the Euclidean norm $\|\cdot\|_{2}$. The notations $O(\cdot)$ and $o(\cdot)$ are used in the usual sense. We denote by $e_{q}$ a vector of length $q$ whose entries are all 1 , that is, $e_{q}=(1,1, \ldots, 1)^{T}$.

\section{Assumptions and Background}

In this section, we summarize some known results concerning constraint qualifications and necessary optimality conditions for MPEC and its elastic-mode 
formulation. We discuss first-order stationarity conditions and constraint qualifications for MPEC (1) in Subsection 2.1 and first- and second-order stationarity conditions for $\mathrm{PF}(c)$ (2) in Subsection 2.2. Subsection 2.3 describes the correspondence between certain first-order points of the elastic form (2) and first-order points of the MPEC (1).

\subsection{Stationarity Conditions and Constraint Qualifications for MPEC}

We start by defining the following active sets at a feasible point $x^{*}$ of MPEC (1):

$$
\begin{gathered}
I_{g} \stackrel{\text { def }}{=}\left\{i \in\{1,2, \ldots, p\} \mid g_{i}\left(x^{*}\right)=0\right\}, \\
I_{G} \stackrel{\text { def }}{=}\left\{i \in\{1,2, \ldots, m\} \mid G_{i}^{T} x^{*}=0\right\}, \\
I_{H} \stackrel{\text { def }}{=}\left\{i \in\{1,2, \ldots, m\} \mid H_{i}^{T} x^{*}=0\right\},
\end{gathered}
$$

where $G_{i}$ and $H_{i}$ denote the $i$ th column of $G$ and $H$, respectively (in each case, a column from the identity matrix). Because $x^{*}$ is feasible for (1), we have $I_{G} \cup I_{H}=\{1,2, \ldots, m\}$.

Using the active sets, we define our first notion of first-order stationarity for (1) as follows.

Definition 1. A feasible point $x^{*}$ of (1) is strongly stationary if $d=0$ solves the following linear program:

$$
\begin{gathered}
\min _{d} \nabla f\left(x^{*}\right)^{T} d \quad \text { subject to } \\
g\left(x^{*}\right)+\nabla g\left(x^{*}\right)^{T} d \geq 0, \quad h\left(x^{*}\right)+\nabla h\left(x^{*}\right)^{T} d=0, \\
G_{i}^{T} d=0, \quad i \in I_{G} \backslash I_{H}, \\
H_{i}^{T} d=0, \quad i \in I_{H} \backslash I_{G}, \\
G_{i}^{T} d \geq 0, \quad H_{i}^{T} d \geq 0, \quad i \in I_{G} \cap I_{H} .
\end{gathered}
$$

Let us introduce Lagrange multipliers and define the MPEC Lagrangian as in Scholtes [23, Sec. 4]:

$$
L(x, \lambda, \mu, \tau, \nu)=f(x)-\lambda^{T} g(x)-\mu^{T} h(x)-\tau^{T} G^{T} x-\nu^{T} H^{T} x .
$$

By combining the (necessary and sufficient) conditions for $d=0$ to solve (4) with the feasibility conditions for $x^{*}$, we see that $x^{*}$ is strongly stationary if and only if $x^{*}$ satisfies, together with some multipliers $\left(\lambda^{*}, \mu^{*}, \tau^{*}, \nu^{*}\right)$, the following conditions:

$$
\begin{aligned}
\nabla_{x} L\left(x^{*}, \lambda^{*}, \mu^{*}, \tau^{*}, \nu^{*}\right) & =0, \\
0 \leq \lambda^{*} \perp g\left(x^{*}\right) & \geq 0, \\
h\left(x^{*}\right) & =0, \\
\tau^{*} \perp G^{T} x^{*} & \geq 0, \\
\nu^{*} \perp H^{T} x^{*} & \geq 0, \\
\tau_{i}^{*} & \geq 0, \quad i \in I_{G} \cap I_{H}, \\
\nu_{i}^{*} & \geq 0, \quad i \in I_{G} \cap I_{H} .
\end{aligned}
$$


Under the following constraint qualification at $x^{*}$, the multipliers $\left(\lambda^{*}, \mu^{*}, \tau^{*}, \nu^{*}\right)$ are in fact unique.

Definition 2. The MPEC-LICQ holds at a feasible point $x^{*}$ of (1) if the following set of vectors is linearly independent:

$$
\mathcal{K} \stackrel{\text { def }}{=}\left\{\nabla g_{i}\left(x^{*}\right)\right\}_{i \in I_{g}} \cup\left\{\nabla h_{i}\left(x^{*}\right)\right\}_{i=1,2, \ldots, q} \cup\left\{G_{i}\right\}_{i \in I_{G}} \cup\left\{H_{i}\right\}_{i \in I_{H}} .
$$

The following result, dating back to Luo, Pang, and Ralph [16] but stated here in the form of Scheel and Scholtes [22, Theorem 2], shows that, under MPEC-LICQ, strong stationarity is a set of (first-order) necessary optimality conditions for the MPEC.

Theorem 1. Suppose that $x^{*}$ is a local minimizer of (1). If the MPEC-LICQ holds at $x^{*}$, then $x^{*}$ is strongly stationary, and the multiplier vector $\left(\lambda^{*}, \mu^{*}, \tau^{*}, \nu^{*}\right)$ that satisfies the conditions (6) is unique.

Our analysis also uses two weaker notions of first-order stationarity for (1) that have been studied in previous works; see, for example, Outrata [17] and Scheel and Scholtes [22].

Definition 3. (a) A point $x^{*}$ is C-stationary if there exist multipliers

$\left(\lambda^{*}, \mu^{*}, \tau^{*}, \nu^{*}\right)$ satisfying (6) except that the conditions (6f), (6g) are replaced by $\tau_{i}^{*} \nu_{i}^{*} \geq 0$, for each $i \in I_{G} \cap I_{H}$.

(b) A point $x^{*}$ is M-stationary if it is C-stationary and if either $\tau_{i}^{*} \geq 0$ or $\nu_{i}^{*} \geq 0$ for each $i \in I_{G} \cap I_{H}$.

Notice that M-stationarity allows such situations as $\tau_{i}^{*}<0$ and $\nu_{i}^{*}=0$ for some $i \in I_{G} \cap I_{H}$ but does not allow the situation $\tau_{i}^{*}<0$ and $\nu_{i}^{*}<0$, which is allowed by $\mathrm{C}$-stationarity. In particular, strongly stationary $\Rightarrow$ M-stationary $\Rightarrow$ C-stationary.

\subsection{Necessary Optimality Conditions for PF(c)}

In this subsection, we discuss the exact and inexact first- and second-order necessary optimality conditions for $\mathrm{PF}(c)$ defined in (2). We start by defining the Lagrangian for this problem as follows:

$$
\begin{aligned}
& L_{c}\left(x, \zeta, \lambda, \mu^{-}, \mu^{+}, \tau, \nu\right)=f(x)+c \zeta+c\left(G^{T} x\right)^{T} H^{T} x-\lambda^{T}\left(g(x)+\zeta e_{p}\right)_{(8)} \\
& -\left(\mu^{+}\right)^{T}\left(\zeta e_{q}-h(x)\right)-\left(\mu^{-}\right)^{T}\left(\zeta e_{q}+h(x)\right)-\tau^{T} G^{T} x-\nu^{T} H^{T} x .
\end{aligned}
$$

The Karush- Kuhn-Tucker first-order necessary optimality conditions for this problem are as follows:

$$
\begin{aligned}
\nabla_{x} L_{c}\left(x, \zeta, \lambda, \mu^{-}, \mu^{+}, \tau, \nu\right) & =0, \\
c-e_{p}^{T} \lambda-e_{q}^{T} \mu^{-}-e_{q}^{T} \mu^{+} & =\pi^{-}-\pi^{+}, \\
0 \leq\left(\pi^{-}, \pi^{+}\right) & \perp(\zeta, \bar{\zeta}-\zeta) \geq 0, \\
0 \leq \lambda & \perp g(x)+\zeta e_{p} \geq 0,
\end{aligned}
$$




$$
\begin{aligned}
0 & \leq \mu^{+} \perp \zeta e_{q}-h(x) \geq 0, \\
0 & \leq \mu^{-} \perp \zeta e_{q}+h(x) \geq 0, \\
0 & \leq \tau \perp G^{T} x \geq 0, \\
& \leq \nu \perp H^{T} x \geq 0 .
\end{aligned}
$$

We call $(x, \zeta)$ satisfying these conditions a first-order point of $\mathrm{PF}(c)$. Since these conditions cannot be satisfied exactly in practice, we consider the following inexact first-order conditions.

Definition 4. We say that $(x, \zeta)$ is an $\epsilon$-first-order point of $P F(c)(\epsilon \geq 0)$ if there exist multipliers $\left(\lambda, \mu^{-}, \mu^{+}, \tau, \nu, \pi^{-}, \pi^{+}\right)$satisfying

$$
\begin{array}{lrr} 
& \left\|\nabla_{x} L_{c}\left(x, \zeta, \lambda, \mu^{-}, \mu^{+}, \tau, \nu\right)\right\|_{\infty} \leq \epsilon, \\
& \left|c-e_{p}^{T} \lambda-e_{q}^{T} \mu^{-}-e_{q}^{T} \mu^{+}-\pi^{-}+\pi^{+}\right| \leq \epsilon, \\
0 \leq\left(\pi^{-}, \pi^{+}\right), & (\zeta, \bar{\zeta}-\zeta) \geq 0, & \zeta \pi^{-}+(\bar{\zeta}-\zeta) \pi^{+} \leq \epsilon, \\
0 \leq \lambda, & g(x)+\zeta e_{p} \geq-\epsilon e_{p}, & \left|\left(g(x)+\zeta e_{p}\right)^{T} \lambda\right| \leq \epsilon, \\
0 \leq \mu^{+}, & \zeta e_{q}-h(x) \geq-\epsilon e_{q}, & \left|\left(\zeta e_{q}-h(x)\right)^{T} \mu^{+}\right| \leq \epsilon, \\
0 \leq \mu^{-}, & \zeta e_{q}+h(x) \geq-\epsilon e_{q}, & \left|\left(\zeta e_{q}+h(x)\right)^{T} \mu^{-}\right| \leq \epsilon, \\
0 \leq \tau, & G^{T} x \geq 0, & \tau^{T} G^{T} x \leq \epsilon, \\
0 \leq \nu, & H^{T} x \geq 0, & \nu^{T} H^{T} x \leq \epsilon .
\end{array}
$$

The conditions (10) are well suited to situations in which $\mathrm{PF}(c)$ is solved by interior-point methods or active-set methods, since such methods can enforce the bound constraints $G^{T} x \geq 0$ and $H^{T} x \geq 0$ explicitly (also the nonnegativity constraints on the multipliers, in the case of interior-point methods), while allowing the constraints involving nonlinear functions to be satisfied inexactly.

We now introduce the notions of approximately active constraints and of exact and inexact second-order (stationary) points of $\mathrm{PF}(c)$.

Definition 5. Given a function $r: \mathbb{R}^{n} \rightarrow \mathbb{R}$, a constraint $r(x) \geq 0$ or $r(x)=0$ of a nonlinear program is $\delta$-active $(\delta \geq 0)$ at a point $\hat{x}$ if $|r(\hat{x})| \leq \delta$. The constraint is active at $\hat{x}$ if $r(\hat{x})=0$.

Definition 6. We say that $(x, \zeta)$ is a second-order point of $P F(c)$ if there exist multipliers $\left(\lambda, \mu^{-}, \mu^{+}, \tau, \nu, \pi^{-}, \pi^{+}\right)$satisfying (9) (so $(x, \zeta)$ is a first-order point of $P F(c))$ and

$$
\tilde{u}^{T} \nabla_{(x, \zeta)(x, \zeta)}^{2} L_{c}\left(x, \zeta, \lambda, \mu^{-}, \mu^{+}, \tau, \nu\right) \tilde{u} \geq 0,
$$

for all $\tilde{u} \in \mathbb{R}^{n+1}$ in the null space of the gradients of all active constraints of (2) at $(x, \zeta)$.

Definition 7. We say that $(x, \zeta)$ is an $(\epsilon, \delta)$-second-order point of $\operatorname{PF}(c)(\epsilon, \delta \geq$ $0)$ if there exist multipliers $\left(\lambda, \mu^{-}, \mu^{+}, \tau, \nu, \pi^{-}, \pi^{+}\right)$satisfying (10) (so $(x, \zeta)$ is an $\epsilon$-first-order point of $P F(c))$ and

$$
\tilde{u}^{T} \nabla_{(x, \zeta)(x, \zeta)}^{2} L_{c}\left(x, \zeta, \lambda, \mu^{-}, \mu^{+}, \tau, \nu\right) \tilde{u} \geq-C\|\tilde{u}\|^{2},
$$

for all $\tilde{u} \in \mathbb{R}^{n+1}$ that are simultaneously in the null space of the gradients of all active bound constraints $\left(G^{T} x \geq 0, H^{T} x \geq 0,0 \leq \zeta \leq \bar{\zeta}\right)$ of (2) at $(x, \zeta)$ and in 
the null space of the gradients of $\delta$-active nonbound constraints $\left(g(x) \geq-\zeta e_{p}\right.$, $\left.\zeta e_{q} \geq h(x) \geq-\zeta e_{q}\right)$ at $(x, \zeta)$. Here $C \geq 0$ is an arbitrary constant independent of $(x, \zeta)$.

We shall see in Subsection 5.3 that the bounded indefiniteness condition given in Definition 7 is numerically easier to verify than the more standard positive semidefiniteness condition (corresponding to $C=0$ ). In particular, when we use an off-the-shelf code to solve $\operatorname{PF}(c)$, we generally have no knowledge and no control of how the active constraints are computed, if they are explicitly computed at all. Hence, it is difficult to check numerically whether the final point output by the code satisfies the positive semidefiniteness condition because this condition is sensitive to the value of the (unknown) tolerance $\delta$. On the other hand, as our numerical experience in Subsection 5.3 suggests, the bounded indefiniteness condition seems fairly insensitive to $\delta$.

\subsection{Relating First-Order Points of the MPEC and the Elastic Form}

The following result identifies certain first-order points of $\mathrm{PF}(c)(2)$ with the strongly stationary points of the MPEC (1).

Theorem 2. If $(x, \zeta)$ is a first-order point of $P F(c)$ and $x$ is feasible for (1), then $x$ is strongly stationary for (1) and $\zeta=0$ whenever $c>0$.

Proof. We show first that either $\zeta=0$ or $\zeta$ can be replaced by zero and the first-order conditions (9) will still hold.

Suppose that $\zeta>0$. Since $x$ is feasible for (1), we have from the complementarity conditions (9d), (9e), and (9f) that $\lambda=0$ and $\mu^{+}=\mu^{-}=0$. It follows that the left-hand side of (9b) equals $c$. If $c>0$, then $\pi^{-}>0$, which by (9c) implies $\zeta=0$, contradicting $\zeta>0$. If $c=0$, then $\pi^{-}=\pi^{+}$, which by (9c) implies $\pi^{-}=\pi^{+}=0$. Hence, we can have $\zeta>0$ only if $c=0, \pi^{-}=\pi^{+}=0, \lambda=0$, and $\mu^{+}=\mu^{-}=0$. In this case, we can replace $\zeta$ by 0 and the conditions (9) will still hold.

With $\zeta=0$ in (9), we establish strong stationarity of $x$ by setting $x^{*}=x$ in (6) and choosing appropriate values for the multipliers $\left(\lambda^{*}, \mu^{*}, \tau^{*}, \nu^{*}\right)$. The relevant choices are as follows:

$$
\tau^{*}=\tau-c H^{T} x, \quad \nu^{*}=\nu-c G^{T} x, \quad \lambda^{*}=\lambda, \quad \mu^{*}=\mu^{-}-\mu^{+} .
$$

It is not difficult to verify from (9) (with $\zeta=0$ ) that these choices will satisfy (6).

\section{Global Convergence Results}

In this section we state and prove results for methods in which $\mathrm{PF}\left(c_{k}\right)$ is solved for a nondecreasing sequence of positive scalars $\left\{c_{k}\right\}$. By "solved" we mean that either an exact or inexact first- or second-order point $x^{k}$ of $\operatorname{PF}\left(c_{k}\right)$ is computed; we analyze various cases in the subsections below. We are interested particularly in techniques that achieve exact complementarity finitely; that is, $\left(G^{T} x_{k}\right)^{T}\left(H^{T} x^{k}\right)=0$ for all iterates $k$ with $c_{k}$ exceeding some threshold $c^{*}$. 


\subsection{A Sequence of Inexact First-Order Points}

Here we consider the situation in which an inexact first-order point $\left(x^{k}, \zeta_{k}\right)$ of $\mathrm{PF}\left(c_{k}\right)$ is generated, for $k=0,1, \ldots$, and give conditions under which accumulation points of $\left\{x^{k}\right\}$ are C-stationary. The proof is long and somewhat technical. It borrows some ideas from the proofs of Scholtes [23, Theorem 3.1] and Anitescu [2, Theorem 2.5]. In particular, we use the inexact first-order conditions (10) of Definition 4 and a technical lemma to deduce, under MPEC-LICQ, the existence of multipliers $\left(\lambda^{*}, \mu^{*}, \tau^{*}, \nu^{*}\right)$ satisfying the first stationarity condition (6a) with each accumulation point $x^{*}$. We then analyze the signs of the multipliers $\tau^{*}$ and $\nu^{*}$ to show that $x^{*}$ is C-stationary. Finally, we show by a contradiction argument that the relevant subsequence $\left\{\zeta_{k}\right\}$ converges to zero.

Theorem 3. Let $\left\{c_{k}\right\}$ be a positive sequence, nondecreasing with $k$, and $\left\{\epsilon_{k}\right\}$ be a nonnegative sequence with $\left\{c_{k} \epsilon_{k}\right\} \rightarrow 0$. Suppose that $\left(x^{k}, \zeta_{k}\right)$ is an $\epsilon_{k}$-firstorder point of $P F\left(c_{k}\right), k=0,1, \ldots$ Let $x^{*}$ be any accumulation point of $\left\{x^{k}\right\}$ that is feasible for (1) and satisfies MPEC-LICQ. Then $x^{*}$ is C-stationary for (1), and for any $\mathcal{S} \subset\{0,1, \ldots\}$ with $\left\{x^{k}\right\}_{k \in \mathcal{S}} \rightarrow x^{*}$, we have $\left\{\zeta_{k}\right\}_{k \in \mathcal{S}} \rightarrow 0$.

Proof. Suppose without loss of generality that $\left\{x^{k}\right\} \rightarrow x^{*}$. Since $c_{k} \geq c_{0}>$ 0 and $\left\{c_{k} \epsilon_{k}\right\} \rightarrow 0$, we have $\left\{\epsilon_{k}\right\} \rightarrow 0$. Let $\left(\lambda^{k}, \mu^{-k}, \mu^{+k}, \tau^{k}, \nu^{k}, \pi^{-k}, \pi^{+k}\right)$ be multipliers associated with $\left(x^{k}, \zeta_{k}\right)$ (from (10)).

From the final row of (10), we have that, for all $k$,

$$
\nu_{i}^{k}\left(H_{i}^{T} x^{k}\right) \leq\left(\nu^{k}\right)^{T}\left(H^{T} x^{k}\right) \leq \epsilon_{k}, \quad i=1,2, \ldots, m,
$$

so for $i \notin I_{H}$, since $H_{i}^{T} x^{k}$ is bounded away from zero, we have that $\nu_{i}^{k}=O\left(\epsilon_{k}\right)$. By similar reasoning, we have that $\tau_{i}^{k}=O\left(\epsilon_{k}\right)$ for $i \notin I_{G}$. Using these two facts, we can write the first row of (10) as follows:

$$
\begin{aligned}
0=\nabla & f\left(x^{k}\right)-\sum_{i=1}^{p} \lambda_{i}^{k} \nabla g_{i}\left(x^{k}\right)-\sum_{i=1}^{q}\left(\mu_{i}^{-k}-\mu_{i}^{+k}\right) \nabla h_{i}\left(x^{k}\right) \\
& -\sum_{i \in I_{G}}\left(\tau_{i}^{k}-c_{k} H_{i}^{T} x^{k}\right) G_{i}-\sum_{i \in I_{H}}\left(\nu_{i}^{k}-c_{k} G_{i}^{T} x^{k}\right) H_{i} \\
& +c_{k} \sum_{i \notin I_{G}}\left(H_{i}^{T} x^{k}\right) G_{i}+c_{k} \sum_{i \notin I_{H}}\left(G_{i}^{T} x^{k}\right) H_{i}+O\left(\epsilon_{k}\right) .
\end{aligned}
$$

Since $x^{*}$ is feasible for (1), we have $I_{G} \cup I_{H}=\{1,2, \ldots, m\}$, and the set of indices $i \notin I_{G}$ is simply $I_{H} \backslash I_{G}$. Similarly, $i \notin I_{H} \Leftrightarrow i \in I_{G} \backslash I_{H}$. Hence, we can restate the relation above as follows:

$$
\begin{aligned}
0=\nabla & f\left(x^{k}\right)-\sum_{i=1}^{p} \lambda_{i}^{k} \nabla g_{i}\left(x^{k}\right)-\sum_{i=1}^{q}\left(\mu_{i}^{-k}-\mu_{i}^{+k}\right) \nabla h_{i}\left(x^{k}\right) \\
& -\sum_{i \in I_{G} \cap I_{H}}\left(\tau_{i}^{k}-c_{k} H_{i}^{T} x^{k}\right) G_{i}-\sum_{i \in I_{G} \cap I_{H}}\left(\nu_{i}^{k}-c_{k} G_{i}^{T} x^{k}\right) H_{i}
\end{aligned}
$$




$$
\begin{aligned}
& -\sum_{i \in I_{G} \backslash I_{H}}\left[\left(\tau_{i}^{k}-c_{k} H_{i}^{T} x^{k}\right) G_{i}-c_{k}\left(G_{i}^{T} x^{k}\right) H_{i}\right] \\
& -\sum_{i \in I_{H} \backslash I_{G}}\left[\left(\nu_{i}^{k}-c_{k} G_{i}^{T} x^{k}\right) H_{i}-c_{k}\left(H_{i}^{T} x^{k}\right) G_{i}\right]+O\left(\epsilon_{k}\right) .
\end{aligned}
$$

We examine the final summation in (13) more closely. This term can be written as follows:

$$
\begin{aligned}
& \sum_{i \in I_{H} \backslash I_{G}}\left[\left(\nu_{i}^{k}-c_{k} G_{i}^{T} x^{k}\right) H_{i}-c_{k}\left(H_{i}^{T} x^{k}\right) G_{i}\right] \\
= & \sum_{i \in I_{H} \backslash I_{G}}\left(\nu_{i}^{k}-c_{k} G_{i}^{T} x^{k}\right)\left[H_{i}+\frac{H_{i}^{T} x^{k}}{G_{i}^{T} x^{k}} G_{i}\right]-\nu_{i}^{k} \frac{H_{i}^{T} x^{k}}{G_{i}^{T} x^{k}} G_{i} \\
= & \sum_{i \in I_{H} \backslash I_{G}}\left(\nu_{i}^{k}-c_{k} G_{i}^{T} x^{k}\right)\left[H_{i}+\frac{H_{i}^{T} x^{k}}{G_{i}^{T} x^{k}} G_{i}\right]+O\left(\epsilon_{k}\right),
\end{aligned}
$$

where the final inequality is a consequence of $\left\{G_{i}^{T} x^{k}\right\} \rightarrow G_{i}^{T} x^{*}>0$ for $i \in I_{H} \backslash I_{G}$ and $0 \leq \nu_{i}^{k} H_{i}^{T} x^{k} \leq \epsilon_{k}$ (see (12)). Hence, by defining

$$
\tilde{H}_{i}^{k} \stackrel{\text { def }}{=} \begin{cases}H_{i}+\frac{H_{i}^{T} x^{k}}{G_{i}^{T} x^{k}} G_{i}, & \text { for } i \in I_{H} \backslash I_{G}, \\ H_{i}, & \text { for } i \in I_{G} \cap I_{H},\end{cases}
$$

we deduce from (14) that

$$
\sum_{i \in I_{H} \backslash I_{G}}\left[\left(\nu_{i}^{k}-c_{k} G_{i}^{T} x^{k}\right) H_{i}-c_{k}\left(H_{i}^{T} x^{k}\right) G_{i}\right]=\sum_{i \in I_{H} \backslash I_{G}}\left(\nu_{i}^{k}-c_{k} G_{i}^{T} x^{k}\right) \tilde{H}_{i}^{k}+O\left(\epsilon_{k}\right) .
$$

Since $\left\{H_{i}^{T} x^{k} / G_{i}^{T} x^{k}\right\} \rightarrow 0$ for $i \in I_{H} \backslash I_{G}$, we have from (15) that

$$
\left\{\tilde{H}_{i}^{k}\right\} \rightarrow H_{i}, \text { for } i \in I_{H} \text {. }
$$

A similar definition of $\tilde{G}_{i}^{k}$ for $i \in I_{G}$ yields for the second-to-last summation in (13) that

$$
\sum_{i \in I_{G} \backslash I_{H}}\left[\left(\tau_{i}^{k}-c_{k} H_{i}^{T} x^{k}\right) G_{i}-c_{k}\left(G_{i}^{T} x^{k}\right) H_{i}\right]=\sum_{i \in I_{G} \backslash I_{H}}\left(\tau_{i}^{k}-c_{k} H_{i}^{T} x^{k}\right) \tilde{G}_{i}^{k}+O\left(\epsilon_{k}\right) .
$$

By substituting (16) and (17) into (13) and using the definitions of $\tilde{H}_{i}^{k}$ and $\tilde{G}_{i}^{k}$, we have

$$
\begin{aligned}
0=\nabla & f\left(x^{k}\right)-\sum_{i=1}^{p} \lambda_{i}^{k} \nabla g_{i}\left(x^{k}\right)-\sum_{i=1}^{q}\left(\mu_{i}^{-k}-\mu_{i}^{+k}\right) \nabla h_{i}\left(x^{k}\right) \\
& -\sum_{i \in I_{G}}\left(\tau_{i}^{k}-c_{k} H_{i}^{T} x^{k}\right) \tilde{G}_{i}^{k}-\sum_{i \in I_{H}}\left(\nu_{i}^{k}-c_{k} G_{i}^{T} x^{k}\right) \tilde{H}_{i}^{k}+O\left(\epsilon_{k}\right) .
\end{aligned}
$$

We turn now to the term in (18) involving $\lambda^{k}$. By taking a further subsequence if necessary, we assume that there is a constant $\rho>0$ such that 
$g_{i}\left(x^{k}\right) \geq \rho$ for all $i \notin I_{g}$ and all $k$. From the fourth row of (10) we have $\left|\left(g\left(x^{k}\right)+\zeta_{k} e_{p}\right)^{T} \lambda^{k}\right| \leq \epsilon_{k}$ and therefore

$$
\sum_{i \notin I_{g}}\left(g_{i}\left(x^{k}\right)+\zeta_{k}\right) \lambda_{i}^{k} \leq \epsilon_{k}-\sum_{i \in I_{g}}\left(g_{i}\left(x^{k}\right)+\zeta_{k}\right) \lambda_{i}^{k} \leq \epsilon_{k}+\epsilon_{k} \sum_{i \in I_{g}} \lambda_{i}^{k},
$$

where the second inequality follows from the fact that $\lambda_{i}^{k} \geq 0$ and $g_{i}\left(x^{k}\right)+\zeta_{k} \geq$ $-\epsilon_{k}$ for all $i$ (due to the fourth row of (10)). Since $i \notin I_{g} \Rightarrow g_{i}\left(x^{k}\right)+\zeta_{k} \geq$ $g_{i}\left(x^{k}\right) \geq \rho>0$, it follows that

$$
\rho \sum_{i \notin I_{g}} \lambda_{i}^{k} \leq \epsilon_{k}+\epsilon_{k} \sum_{i \in I_{g}} \lambda_{i}^{k}, \text { for all } k .
$$

When $\sum_{i \in I_{g}} \lambda_{i}^{k} \geq 1$, we have immediately from (19) that

$$
\frac{\sum_{i \notin I_{g}} \lambda_{i}^{k}}{\sum_{i \in I_{g}} \lambda_{i}^{k}} \leq \frac{2 \epsilon_{k}}{\rho}
$$

Then

$$
\sum_{i=1}^{p} \lambda_{i}^{k} \nabla g_{i}\left(x^{k}\right)=\sum_{i \in I_{g}} \lambda_{i}^{k}\left[\nabla g_{i}\left(x^{k}\right)+\frac{\sum_{j \notin I_{g}} \lambda_{j}^{k} \nabla g_{j}\left(x^{k}\right)}{\sum_{j \in I_{g}} \lambda_{j}^{k}}\right]=\sum_{i \in I_{g}} \lambda_{i}^{k} \tilde{g}_{i},
$$

where the vector $\tilde{g}_{i}^{k}$ is defined in the obvious way. Because of $(20)$ and $\left\{x^{k}\right\} \rightarrow x^{*}$, we have $\left\{\tilde{g}_{i}^{k}\right\} \rightarrow \nabla g_{i}\left(x^{*}\right)$. Otherwise, when $\sum_{i \in I_{g}} \lambda_{i}^{k}<1$, we have from (19) that

$$
\sum_{i \notin I_{g}} \lambda_{i}^{k} \leq \frac{2 \epsilon_{k}}{\rho}=O\left(\epsilon_{k}\right)
$$

so that

$$
\sum_{i=1}^{p} \lambda_{i}^{k} \nabla g_{i}\left(x^{k}\right)=\sum_{i \in I_{g}} \lambda_{i}^{k} \tilde{g}_{i}^{k}+O\left(\epsilon_{k}\right)
$$

where we set $\tilde{g}_{i}^{k} \stackrel{\text { def }}{=} \nabla g_{i}\left(x^{k}\right)$. Thus, in both cases, we have that

$$
\sum_{i=1}^{p} \lambda_{i}^{k} \nabla g_{i}\left(x^{k}\right)=\sum_{i \in I_{g}} \lambda_{i}^{k} \tilde{g}_{i}^{k}+O\left(\epsilon_{k}\right) \quad \text { and } \quad\left\{\tilde{g}_{i}^{k}\right\} \rightarrow \nabla g_{i}\left(x^{*}\right), i \in I_{g} .
$$

Using the first relation, we can write (18) as follows:

$$
\begin{aligned}
0=\nabla & f\left(x^{k}\right)-\sum_{i \in I_{g}} \lambda_{i}^{k} \tilde{g}_{i}^{k}-\sum_{i=1}^{q}\left(\mu_{i}^{-k}-\mu_{i}^{+k}\right) \nabla h_{i}\left(x^{k}\right) \\
& -\sum_{i \in I_{G}}\left(\tau_{i}^{k}-c_{k} H_{i}^{T} x^{k}\right) \tilde{G}_{i}^{k}-\sum_{i \in I_{H}}\left(\nu_{i}^{k}-c_{k} G_{i}^{T} x^{k}\right) \tilde{H}_{i}^{k}+O\left(\epsilon_{k}\right) .
\end{aligned}
$$


Since $x^{*}$ satisfies MPEC-LICQ, we can invoke Lemma 2 (see the appendix) to deduce from (22) the existence of $\lambda_{i}^{*}$ for $i \in I_{g}, \tau_{i}^{*}$ for $i \in I_{G}$, and $\nu_{i}^{*}$ for $i \in I_{H}$ such that

$$
0=\nabla f\left(x^{*}\right)-\sum_{i \in I_{g}} \lambda_{i}^{*} \nabla g_{i}\left(x^{*}\right)-\sum_{i=1}^{q} \mu_{i}^{*} \nabla h_{i}\left(x^{*}\right)-\sum_{i \in I_{G}} \tau_{i}^{*} G_{i}-\sum_{i \in I_{H}} \nu_{i}^{*} H_{i},
$$

and, moreover,

$$
\begin{aligned}
\left\{\lambda_{i}^{k}\right\} & \rightarrow \lambda_{i}^{*}, \quad \text { for } i \in I_{g}, \\
\left\{\mu_{i}^{-k}-\mu_{i}^{+k}\right\} & \rightarrow \mu_{i}^{*}, \quad \text { for } i=1,2, \ldots, q, \\
\left\{\tau_{i}^{k}-c_{k} H_{i}^{T} x^{k}\right\} & \rightarrow \tau_{i}^{*}, \quad \text { for } i \in I_{G}, \\
\left\{\nu_{i}^{k}-c_{k} G_{i}^{T} x^{k}\right\} & \rightarrow \nu_{i}^{*}, \quad \text { for } i \in I_{H} .
\end{aligned}
$$

We now analyze (23c) and (23d) for $i \in I_{G} \cap I_{H}$. Since $\tau_{i}^{k}, \nu_{i}^{k}, G_{i}^{T} x^{k}$, and $H_{i}^{T} x^{k}$ are all nonnegative, we have

$$
\begin{aligned}
& \left(\tau_{i}^{k}-c_{k} H_{i}^{T} x^{k}\right)\left(\nu_{i}^{k}-c_{k} G_{i}^{T} x^{k}\right) \\
& =\tau_{i}^{k} \nu_{i}^{k}+c_{k}^{2}\left(H_{i}^{T} x^{k}\right)\left(G_{i}^{T} x^{k}\right)-c_{k}\left(\tau_{i}^{k} G_{i}^{T} x^{k}+\nu_{i}^{k} H_{i}^{T} x^{k}\right) \\
& \geq-c_{k}\left(\tau_{i}^{k} G_{i}^{T} x^{k}+\nu_{i}^{k} H_{i}^{T} x^{k}\right) \\
& \geq-2 c_{k} \epsilon_{k},
\end{aligned}
$$

where the final inequality follows from (10). Taking limits as $k \rightarrow \infty$ and using $\left\{c_{k} \epsilon_{k}\right\} \rightarrow 0$, we conclude that $\tau_{i}^{*} \nu_{i}^{*} \geq 0$ for $i \in I_{G} \cap I_{H}$, implying C-stationarity.

To complete the proof, we show by contradiction that $\left\{\zeta_{k}\right\} \rightarrow 0$. If this limit did not hold, we could assume by taking a subsequence if necessary that $\zeta_{k} \geq \underline{\zeta}>0$ for all $k$. Since $x^{*}$ is feasible, we have that $g_{i}\left(x^{*}\right) \geq 0$ for all $i$, so for all $k$ sufficiently large we have

$$
g_{i}\left(x^{k}\right)+\zeta_{k} \geq \underline{\zeta} / 2, \quad \text { for } i=1,2, \ldots, p .
$$

Hence, we have from the fourth row of (10) that

$$
e_{p}^{T} \lambda^{k} \leq 2 \epsilon_{k} / \underline{\zeta},
$$

for all $k$ sufficiently large. Similarly, since $h\left(x^{*}\right)=0$, we have that

$$
\zeta_{k}-h_{i}\left(x^{k}\right) \geq \underline{\zeta} / 2, \quad \zeta_{k}+h_{i}\left(x^{k}\right) \geq \underline{\zeta} / 2, \quad \text { for } i=1,2, \ldots, q .
$$

Hence

$$
e_{q}^{T} \mu^{-k} \leq 2 \epsilon_{k} / \underline{\zeta}, \quad e_{q}^{T} \mu^{+k} \leq 2 \epsilon_{k} / \underline{\zeta},
$$

for all $k$ sufficiently large. This together with the second row of (10) yields

$$
\pi^{-k}-\pi^{+k}=c^{k}+O\left(\epsilon^{k}\right) .
$$

Since $\pi^{+k} \geq 0$, this implies $\pi^{-k} \geq c^{k}+O\left(\epsilon^{k}\right)$. Also, from the third row of (10), we have $\zeta_{k} \pi^{-k} \leq \epsilon_{k}$. Thus

$$
\zeta_{k} \leq \frac{\epsilon_{k}}{\pi^{-k}} \leq \frac{\epsilon_{k}}{c_{k}+O\left(\epsilon_{k}\right)} \rightarrow 0 \text { as } k \rightarrow \infty
$$


contradicting our positive lower bound on $\zeta_{k}$. $\square$

Without loss of generality, we could assume in Theorem 3 that $\left\{c_{k}\right\}$ is increasing (rather than nondecreasing). However, allowing $\left\{c_{k}\right\}$ to be nondecreasing is convenient when, for example, $\left(x^{k}, \zeta_{k}\right)$ is the point generated at the $k$ th iteration of an iterative method that allows $c_{k}$ to remain unchanged from one iteration to the next; see Algorithm Elastic-Inexact in Section 3.5.

The following corollary gives additional global convergence properties of the sequence $\left\{\left(x^{k}, \zeta_{k}\right)\right\}$.

Corollary 1. Suppose that the assumptions of Theorem 3 hold, where $x^{*}$ is an accumulation point of $\left\{x^{k}\right\}$ that is C-stationary for (1) and satisfies MPEC$L I C Q$. Then for any $\mathcal{S} \subset\{0,1, \ldots\}$ such that $\left\{x^{k}\right\}_{k \in \mathcal{S}} \rightarrow x^{*}$, we have that

$$
\begin{aligned}
\left\{c_{k} G_{i}^{T} x^{k}\right\}_{k \in \mathcal{S}} & \rightarrow 0, \text { for } i \in I_{G} \backslash I_{H}, \\
\left\{c_{k} H_{i}^{T} x^{k}\right\}_{k \in \mathcal{S}} & \rightarrow 0, \text { for } i \in I_{H} \backslash I_{G}, \\
\left\{c_{k}\left(G_{i}^{T} x^{k}\right)\left(H_{i}^{T} x^{k}\right)\right\}_{k \in \mathcal{S}} & \rightarrow 0, \text { for } i \in I_{G} \cap I_{H}, \\
\left\{c_{k} \zeta_{k}\right\}_{k \in \mathcal{S}} & \rightarrow 0 .
\end{aligned}
$$

Proof. We first prove (24b); the proof of (24a) is analogous. If $\left\{c_{k}\right\}$ is bounded (from above by $\bar{c}$, say), then the result follows from

$$
0 \leq c_{k} H_{i}^{T} x^{k} \leq \bar{c} H_{i}^{T} x^{k} \rightarrow \bar{c} H_{i}^{T} x^{*}=0, \quad \text { as } k \in \mathcal{S}, k \rightarrow \infty, \quad i \in I_{H} \backslash I_{G} .
$$

Suppose instead that $\left\{c_{k}\right\} \uparrow \infty$. Assume for contradiction that there is some $\overline{\mathcal{S}} \subset \mathcal{S}$, some $i \in I_{H} \backslash I_{G}$, and some constant $\rho>0$ such that $c_{k} H_{i}^{T} x^{k} \geq \rho$ for all $k \in \overline{\mathcal{S}}$. From the final row of (10), we have that $\nu_{i}^{k} H_{i}^{T} x^{k} \leq\left(\nu^{k}\right)^{T} H^{T} x^{k} \leq \epsilon_{k}$, implying

$$
\nu_{i}^{k} c_{k} H_{i}^{T} x^{k} \leq c_{k} \epsilon_{k} \rightarrow 0, \quad \text { as } k \in \overline{\mathcal{S}}, k \rightarrow \infty .
$$

It follows from $c_{k} H_{i}^{T} x^{k} \geq \rho$ that $\left\{\nu_{i}^{k}\right\}_{k \in \overline{\mathcal{S}}} \rightarrow 0$. From the limit (23d), we then have that

$$
\left\{c_{k} G_{i}^{T} x^{k}\right\}_{k \in \overline{\mathcal{S}}} \rightarrow-\nu_{i}^{*} .
$$

Since $\left\{c_{k}\right\} \uparrow \infty$, this limit implies that $\left\{G_{i}^{T} x^{k}\right\}_{k \in \overline{\mathcal{S}}} \rightarrow 0$. Since $\left\{x^{k}\right\} \rightarrow x^{*}$, it follows that $G_{i}^{T} x^{*}=0$, implying that $i \in I_{G}$. This contradicts our choice of $i \in I_{H} \backslash I_{G}$, so (24b) must hold in this case too.

If $\left\{c_{k}\right\}$ is bounded, then (24c) follows from the feasibility of $x^{*}$ for (1). Suppose instead that $\left\{c_{k}\right\} \uparrow \infty$. Assume for contradiction that there is some $\overline{\mathcal{S}} \subset \mathcal{S}$, some $i \in I_{G} \cap I_{H}$, and some constant $\rho>0$ such that $c_{k}\left(G_{i}^{T} x^{k}\right)\left(H_{i}^{T} x^{k}\right) \geq \rho$ for all $k \in \overline{\mathcal{S}}$. Thus by (23c), we have

$$
\tau_{i}^{k}=c_{k} H_{i}^{T} x^{k}+O(1) \geq \frac{\rho}{G_{i}^{T} x^{k}}+O(1) \geq \frac{\rho}{2 G_{i}^{T} x^{k}},
$$

for all $k \in \overline{\mathcal{S}}$ sufficiently large. However, from the second-to-last row of (10), we have $\tau_{i}^{k} G_{i}^{T} x^{k} \leq\left(\tau^{k}\right)^{T}\left(G^{T} x^{k}\right) \leq \epsilon_{k}$, so that $\tau_{i}^{k} \leq \epsilon_{k} / G_{i}^{T} x^{k}$, yielding the desired contradiction since $\left\{\epsilon_{k}\right\} \rightarrow 0$.

To prove (24d), we see from the third row of (10) that, for all $k$,

$$
c_{k} \zeta_{k} \leq \zeta_{k}\left(e_{p}^{T} \lambda^{k}+e_{q}^{T} \mu^{-k}+e_{q}^{T} \mu^{+k}+\pi^{-k}-\pi^{+k}\right)+\epsilon_{k} \zeta_{k} .
$$


Because of (23a) and $\left\{\zeta_{k}\right\}_{k \in \mathcal{S}} \rightarrow 0$ (Theorem 3), we have that $\left\{\zeta_{k} e_{p}^{T} \lambda^{k}\right\}_{k \in \mathcal{S}} \rightarrow 0$. Similarly, it is immediate that $\left\{\epsilon_{k} \zeta_{k}\right\}_{k \in \mathcal{S}} \rightarrow 0$. From the fifth and sixth rows of (10) and (23b), we also have

$$
\begin{aligned}
& \zeta_{k}\left(e_{q}^{T} \mu^{+k}+e_{q}^{T} \mu^{-k}\right) \\
& \leq h\left(x^{k}\right)^{T}\left(\mu^{+k}-\mu^{-k}\right)+2 \epsilon_{k} \\
& \leq\left\|h\left(x^{k}\right)\right\|_{\infty}\left\|\mu^{+k}-\mu^{-k}\right\|_{1}+2 \epsilon_{k} \\
& \leq\left(\zeta_{k}+\epsilon_{k}\right)\left\|\mu^{+k}-\mu^{-k}\right\|_{1}+2 \epsilon_{k} \rightarrow 0, \quad \text { as } k \in \mathcal{S}, k \rightarrow \infty .
\end{aligned}
$$

Lastly, from the third row of (10), we have $\zeta_{k}\left(\pi^{-k}-\pi^{+k}\right) \leq \epsilon_{k}-\bar{\zeta} \pi^{+k} \leq \epsilon_{k}$. Hence, by taking limits in (25), we have the desired result $(24 \mathrm{~d})$.

In Theorem 3, we assumed that the accumulation point $x^{*}$ is feasible for (1). This assumption is fairly mild and, as we show below, is satisfied under the following assumptions on $\left\{\left(x^{k}, \zeta_{k}\right)\right\}$ and $\left\{c_{k}\right\}$.

Assumption 1 (a) $\left\{f\left(x^{k}\right)\right\}$ is bounded from below.

(b) $\left\{f\left(x^{k}\right)+c_{k} \zeta_{k}+c_{k}\left(G^{T} x^{k}\right)^{T}\left(H^{T} x^{k}\right)\right\}$ is bounded from above.

(c) There exist positive sequences $\left\{\omega_{k}\right\} \rightarrow 0,\left\{\eta_{k}\right\} \rightarrow \infty$ such that $c_{k+1} \geq \eta_{k+1}$ whenever $\zeta_{k}+\left(G^{T} x^{k}\right)^{T}\left(H^{T} x^{k}\right) \geq \omega_{k}$.

Assumption 1(a) holds if $f$ is bounded from below over the feasible set of $\mathrm{PF}\left(c_{k}\right)$. Assumption 1(b) holds if (i) the method for solving $\mathrm{PF}\left(c_{k}\right)$ has the property that the final point $\left(x^{k}, \zeta_{k}\right)$ it generates has objective value no greater than that of the starting point whenever the starting point is feasible for $\operatorname{PF}\left(c_{k}\right)$; and (ii) this method is started at $(\bar{x}, 0)$, with $\bar{x}$ a feasible point of (1). Then $(\bar{x}, 0)$ is feasible for $\operatorname{PF}\left(c_{k}\right)$, with objective value $f(\bar{x})$, so that

$$
f\left(x^{k}\right)+c_{k} \zeta_{k}+c_{k}\left(G^{T} x^{k}\right)^{T}\left(H^{T} x^{k}\right) \leq f(\bar{x}), \text { for all } k .
$$

Assumption 1(c) holds if we choose $c_{k+1} \geq \max \left\{c_{k}, \eta_{k+1}\right\}$ whenever $\left(G^{T} x^{k}\right)^{T}\left(H^{T} x^{k}\right)+\zeta_{k} \geq \omega_{k}$. Assumption 1 contrasts with the infeasible-point MPEC-LICQ assumption used in [9, Lemma 3.2].

Lemma 1. Let $\left\{c_{k}\right\}$ be a positive sequence, nondecreasing with $k$, and $\left\{\epsilon_{k}\right\}$ be a nonnegative sequence with $\left\{\epsilon_{k}\right\} \rightarrow 0$. Suppose that $\left(x^{k}, \zeta_{k}\right)$ is an $\epsilon_{k}$-first-order point of $P F\left(c_{k}\right), k=0,1, \ldots$, and that Assumption 1 is satisfied. Then every accumulation point of $\left\{x^{k}\right\}$ is feasible for (1).

Proof. It suffices to show that

$$
\left\{\zeta_{k}\right\} \rightarrow 0, \quad\left\{\left(G^{T} x^{k}\right)^{T}\left(H^{T} x^{k}\right)\right\} \rightarrow 0
$$

Then any accumulation point $\left(x^{*}, \zeta_{*}\right)$ of $\left\{\left(x^{k}, \zeta_{k}\right)\right\}$ satisfies $\left(G^{T} x^{*}\right)^{T}\left(H^{T} x^{*}\right)=0$ and $\zeta_{*}=0$, implying that $x^{*}$ is feasible for (1). (The other constraints of $(1)$ are satisfied by $x^{*}$, from rows 4 to 8 of $(10)$ and $\left\{\epsilon_{k}\right\} \rightarrow 0$.)

We divide our argument into two cases. First, suppose that

$$
\zeta_{k}+\left(G^{T} x^{k}\right)^{T}\left(H^{T} x^{k}\right)<\omega_{k},
$$


for all $k$ sufficiently large. Since $\zeta_{k} \geq 0, G^{T} x^{k} \geq 0, H^{T} x^{k} \geq 0$ for all $k$ and $\left\{\omega_{k}\right\} \rightarrow 0$, the bound (27) implies (26). Second, suppose that (27) fails to hold for all $k$ in some infinite subsequence. Then, by Assumption 1(c), $c_{k+1} \geq \eta_{k+1}$ for all $k$ in this subsequence. Since $\left\{c_{k}\right\}$ is nondecreasing and $\left\{\eta_{k}\right\} \rightarrow \infty$, we have that $\left\{c_{k}\right\} \uparrow \infty$. Assumptions 1(a) and 1(b) imply that $\left\{c_{k} \zeta_{k}+c_{k}\left(G^{T} x^{k}\right)^{T}\left(H^{T} x^{k}\right)\right\}$ is bounded from above. Since $\zeta_{k} \geq 0, G^{T} x^{k} \geq 0, H^{T} x^{k} \geq 0$ for all $k$ and $\left\{c_{k}\right\} \uparrow \infty$, (26) follows.

\subsection{A Sequence of Exact Second-Order Points}

In this subsection, we consider the situation in which an exact second-order point $\left(x^{k}, \zeta_{k}\right)$ of $\mathrm{PF}\left(c_{k}\right)$ is generated (Definition 6 ), for $k=0,1, \ldots$, with $\left\{c_{k}\right\} \uparrow \infty$.

\section{Algorithm Elastic-Exact}

Choose $c_{k}>0, k=0,1, \ldots$, with $\left\{c_{k}\right\} \uparrow \infty$;

for $k=0,1,2 \ldots$

Find a second-order point $\left(x^{k}, \zeta_{k}\right)$ of $\mathrm{PF}\left(c_{k}\right)$ with Lagrange multipliers $\left(\lambda^{k}, \mu^{-k}, \mu^{+k}, \tau^{k}, \nu^{k}, \pi^{-k}, \pi^{+k}\right)$;

if $\zeta_{k}=0$ and $\left(G^{T} x^{k}\right)^{T}\left(H^{T} x^{k}\right)=0$,

STOP.

end (for)

end (if)

We show below that either the algorithm terminates finitely - in which case, the final iterate $x^{k}$ is strongly stationary by Theorem 2 - or each accumulation point of $\left\{x^{k}\right\}$ either is infeasible or fails to satisfy MPEC-LICQ.

Theorem 4. If Algorithm Elastic-Exact does not terminate finitely, then every accumulation point $x^{*}$ of $\left\{x^{k}\right\}$ either is infeasible for (1) or fails to satisfy $M P E C-L I C Q$.

Proof. Assume for contradiction that the algorithm does not terminate finitely and that there is an accumulation point $x^{*}$ that is feasible for (1) and satisfies MPEC-LICQ. Let $\mathcal{S} \subset\{0,1, \ldots\}$ index the subsequence for which $\left\{x^{k}\right\}_{k \in \mathcal{S}} \rightarrow x^{*}$. Since $\left(x^{k}, \zeta_{k}\right)$ is a first-order point of $\mathrm{PF}\left(c_{k}\right)$, with multipliers $\left(\lambda^{k}, \mu^{-k}, \mu^{+k}, \tau^{k}, \nu^{k}\right.$, $\pi^{-k}, \pi^{+k}$ ), Theorem 3 (with $\epsilon_{k} \equiv 0$ ) shows that $x^{*}$ is C-stationary. Our aim is to show that in fact $x^{k}$ is feasible for (1) for sufficiently large $k \in \mathcal{S}$, and hence the algorithm terminates finitely.

For any $k$, if $\zeta_{k}>0$, then (9c) would imply that $\pi^{-k}=0$, and hence

$$
c_{k}-e_{p}^{T} \lambda^{k}-e_{q}^{T}\left(\mu^{-k}+\mu^{+k}\right)=-\pi^{+k} \leq 0 .
$$

Moreover, (9e) and (9f) would imply that, for each $i$, either $\mu_{i}^{-k}=0$ or $\mu_{i}^{+k}=$ 0 , and hence $\left\|\mu^{-k}+\mu^{+k}\right\|=\left\|\mu^{-k}-\mu^{+k}\right\|$. Since (23a) and (23b) hold when restricted to $k \in \mathcal{S}$, and since $\left\{c_{k}\right\} \rightarrow \infty$, we have that (28) cannot hold for all $k \in \mathcal{S}$ sufficiently large. Thus, we have

$$
\zeta_{k}=0, \quad \text { for all } k \in \mathcal{S} \text { sufficiently large. }
$$


We next show that because $\left(x^{k}, \zeta_{k}\right)$ satisfies the second-order necessary optimality condition for $\operatorname{PF}\left(c_{k}\right)$, we must have $\left(G_{j}^{T} x^{k}\right)\left(H_{j}^{T} x^{k}\right)=0$ for all $j \in I_{G} \cap I_{H}$ and all $k \in \mathcal{S}$ sufficiently large. Suppose not. By passing to a further subsequence if necessary, there must be an index $j \in I_{G} \cap I_{H}$ such that $\left(G_{j}^{T} x^{k}\right)\left(H_{j}^{T} x^{k}\right) \neq 0$ for all $k \in \mathcal{S}$. Define a direction $d^{k}$ satisfying the following conditions:

$$
\begin{aligned}
\nabla g_{i}\left(x^{k}\right)^{T} d^{k} & =0, \quad \text { for } i \in I_{g}, \\
\nabla h\left(x^{k}\right)^{T} d^{k} & =0, \\
G_{i}^{T} d^{k} & =0, \quad \text { for } i \in I_{G} \text { with } i \neq j, \\
H_{i}^{T} d^{k} & =0, \quad \text { for } i \in I_{H} \text { with } i \neq j, \\
G_{j}^{T} d^{k} & =1, \\
H_{j}^{T} d^{k} & =-1 .
\end{aligned}
$$

Since $\left\{x^{k}\right\}_{k \in \mathcal{S}} \rightarrow x^{*}$ and MPEC-LICQ holds at $x^{*}$, the gradients in this definition are linearly independent for all $k \in \mathcal{S}$ sufficiently large, in which case $d^{k}$ satisfying these equations is well defined. In fact, we can choose $d^{k}$ so that

$$
\left\|d^{k}\right\|=O(1)
$$

Since $\left\{\left(x^{k}, \zeta_{k}\right)\right\}_{k \in \mathcal{S}} \rightarrow\left(x^{*}, 0\right)$, the set of active constraints of $(2)$ at $\left(x^{k}, \zeta_{k}\right)$ is a subset of the active constraints at $\left(x^{*}, 0\right)$ for all $k \in \mathcal{S}$ sufficiently large, in which case the direction $\left(d^{k}, 0\right)$ lies in the direction set described in Definition 6 corresponding to $c_{k},\left(x^{k}, \zeta_{k}, \lambda^{k}, \mu^{-k}, \mu^{+k}, \tau^{k}, \nu^{k}\right)$. (Notice that the constraints $G_{j}^{T} x \geq 0$ and $H_{j}^{T} x \geq 0$ are not active at $\left(x^{k}, \zeta_{k}\right)$ because $\left(G_{j}^{T} x^{k}\right)\left(H_{j}^{T} x^{k}\right) \neq 0$.) Also, (9d) implies $\lambda_{i}^{k}=0, i \notin I_{g}$, for all $k \in \mathcal{S}$ sufficiently large.

From Definition (8) and using $\lambda_{i}^{k}=0$ for $i \notin I_{g}$, we have

$$
\begin{array}{rl}
\nabla_{x x}^{2} L_{c_{k}}\left(x^{k}, \zeta^{k}, \lambda^{k}, \mu^{+k}, \mu^{-k}, \tau^{k}, \nu^{k}\right)=\nabla^{2} & f\left(x^{k}\right)-\sum_{i \in I_{g}} \lambda_{i}^{k} \nabla^{2} g_{i}\left(x^{k}\right) \\
& -\sum_{i=1}^{q}\left(\mu_{i}^{-k}-\mu_{i}^{+k}\right) \nabla^{2} h_{i}\left(x^{k}\right) \\
& +c_{k} \sum_{i=1}^{m}\left(G_{i} H_{i}^{T}+H_{i} G_{i}^{T}\right),
\end{array}
$$

for all $k \in \mathcal{S}$ sufficiently large. Also, (23a) and (23b) hold when restricted to $k \in \mathcal{S}$, so the first three terms on the right-hand side of the above relation (involving $\nabla^{2} f, \nabla^{2} g_{i}$, and $\nabla^{2} h_{i}$ ) are $O(1)$. Thus, we have that

$$
\begin{aligned}
& {\left[\begin{array}{c}
d^{k} \\
0
\end{array}\right]^{T} \nabla_{(x, \zeta)(x, \zeta)}^{2} L_{c_{k}}\left(x^{k}, \zeta^{k}, \lambda^{k}, \mu^{+k}, \mu^{-k}, \tau^{k}, \nu^{k}\right)\left[\begin{array}{c}
d^{k} \\
0
\end{array}\right]} \\
& =\left(d^{k}\right)^{T} \nabla_{x x}^{2} L_{c_{k}}\left(x^{k}, \zeta^{k}, \lambda^{k}, \mu^{+k}, \mu^{-k}, \tau^{k}, \nu^{k}\right) d^{k} \\
& =O(1)+c_{k} \sum_{i=1}^{m}\left(d^{k}\right)^{T}\left(G_{i} H_{i}^{T}+H_{i} G_{i}^{T}\right) d^{k} \\
& =O(1)-2 c_{k},
\end{aligned}
$$


where the last equality uses $(29)$ and $I_{G} \cup I_{H}=\{1,2, \ldots, m\}$. Since $\left\{c_{k}\right\} \rightarrow \infty$, the right-hand side is negative for all $k \in \mathcal{S}$ sufficiently large, thereby contradicting the assumption that $x^{k}$ is a second-order point of $\operatorname{PF}\left(c_{k}\right)$. Hence, we have $\left(G_{j}^{T} x^{k}\right)\left(H_{j}^{T} x^{k}\right)=0$ for all $j \in I_{G} \cap I_{H}$ and for all $k \in \mathcal{S}$ sufficiently large.

Now consider any $i \in I_{G} \backslash I_{H}$. Since $H_{i}^{T} x^{k}$ is bounded away from 0 and (23c) holds when restricted to $k \in \mathcal{S}$, we see from $\left\{c_{k}\right\} \rightarrow \infty$ that $\tau_{i}^{k}>0$ for all $k \in \mathcal{S}$ sufficiently large, implying that $G_{i}^{T} x^{k}=0$ (see (9g)). Similarly, we have $H_{i}^{T} x^{k}=0$ for all $i \in I_{H} \backslash I_{G}$ and all $k \in \mathcal{S}$ sufficiently large.

We conclude that $x^{k}$ is feasible for (1) for all $k \in \mathcal{S}$ sufficiently large, and so the algorithm terminates finitely, yielding the desired contradiction.

The following corollary summarizes the conditions for finite termination, obtained by combining the results above.

Corollary 2. Suppose that every feasible point of (1) satisfies MPEC-LICQ. If $\left\{c_{k}\right\}$ and $\left\{\left(x^{k}, \zeta_{k}\right)\right\}$ generated by Algorithm Elastic-Exact satisfies Assumption 1(a),(b) and $\left\{x^{k}\right\}$ lies in a bounded set, then the algorithm terminates finitely with a strongly stationary point of (1).

Proof. Since $\left\{c_{k}\right\} \uparrow \infty$, Assumption 1(c) holds. Since $\left\{x^{k}\right\}$ lies in a bounded set, if Algorithm Elastic-Exact does not terminate finitely, then $\left\{x^{k}\right\}$ would have an accumulation point $x^{*}$ which by Lemma 1 is feasible for (1). Then $x^{*}$ would satisfy MPEC-LICQ, contradicting the conclusion of Theorem 4. Thus the algorithm must terminate finitely with an $x^{k}$ that is feasible for (1). By Theorem $2, x^{k}$ is strongly stationary for (1).

\subsection{A Sequence of Inexact Second-Order Points}

In this subsection, we study the convergence properties of a sequence of inexact second-order points of $\mathrm{PF}\left(c_{k}\right)$ as $k \rightarrow \infty$, showing that accumulation points are M-stationary under mild assumptions. If the sequence $\left\{c_{k}\right\}$ is bounded, then the accumulation points are strongly stationary. We show that, when $\operatorname{PF}\left(c_{k}\right)$ is solved by methods that enforce exact complementarity between the constraints $G^{T} x \geq 0$ and $H^{T} x \geq 0$ and their respective multipliers at each iteration (including active-set methods such as filterSQP [4]), the generated point $x^{k}$ will become exactly feasible with respect to the complementarity constraints $G^{T} x \perp H^{T} x$, once $c_{k}$ exceeds a certain threshold.

Theorem 5. Let $\left\{c_{k}\right\},\left\{\epsilon_{k}\right\}$, and $\left\{\delta_{k}\right\}$ be nonnegative sequences with $c_{k}>0$ nondecreasing with $k,\left\{c_{k} \epsilon_{k}\right\} \rightarrow 0$, and $\left\{\delta_{k}\right\} \rightarrow 0$. Suppose that $\left(x^{k}, \zeta_{k}\right)$ is an $\left(\epsilon_{k}, \delta_{k}\right)$-second-order point of $P F\left(c_{k}\right)$, with Lagrange multipliers

$\left(\lambda^{k}, \mu^{-k}, \mu^{+k}, \tau^{k}, \nu^{k}, \pi^{-k}, \pi^{+k}\right), \quad$ for $k=0,1, \ldots$ Let $x^{*}$ be any accumulation point of $\left\{x^{k}\right\}$ that is feasible for (1) and satisfies MPEC-LICQ. Then there is a threshold $c^{*}$ (depending on $x^{*}$ ) such that if $c_{k} \geq c^{*}$ for all $k$ sufficiently large, the following results hold:

(a) $x^{*}$ is M-stationary for (1).

(b) If $\left\{c_{k}\right\}$ is bounded, then $x^{*}$ is strongly stationary for (1). 
(c) Suppose that exact complementarity between bound constraints and their multipliers holds at all iterations (that is, $\tau^{k} \perp G^{T} x^{k}$ and $\nu^{k} \perp H^{T} x^{k}$ for all $k$ ). Let $\mathcal{S} \subset\{0,1, \ldots\}$ be such that $\left\{x^{k}\right\}_{k \in \mathcal{S}} \rightarrow x^{*}$. Then for all $k \in \mathcal{S}$ sufficiently large with $c_{k} \geq c^{*}$, we have $\left(G^{T} x^{k}\right)^{T}\left(H^{T} x^{k}\right)=0$.

Proof. (a) Since $\left(x^{k}, \zeta_{k}\right)$ is an $\epsilon_{k}$-first-order point of $\operatorname{PF}\left(c_{k}\right)$, we have from Theorem 3 that $x^{*}$ is C-stationary, with corresponding multipliers $\left(\lambda^{*}, \mu^{*}, \tau^{*}, \nu^{*}\right)$. Since $c_{k} \geq c_{0}>0$ for all $k$ and $\left\{c_{k} \epsilon_{k}\right\} \rightarrow 0$, we have $\left\{\epsilon_{k}\right\} \rightarrow 0$.

Let $\mathcal{S} \subset\{0,1, \ldots\}$ be such that $\left\{x^{k}\right\}_{k \in \mathcal{S}} \rightarrow x^{*}$. Theorem 3 implies that $\left\{\zeta^{k}\right\}_{k \in \mathcal{S}} \rightarrow 0$. Also, the fourth row of (10) together with (23a) and $\left\{\epsilon_{k}\right\} \rightarrow 0$ imply $\left\{\lambda_{i}^{k}\right\}_{k \in \mathcal{S}} \rightarrow 0, i \notin I_{g}$. Since $\left\{\left(x^{k}, \zeta_{k}\right)\right\}_{k \in \mathcal{S}} \rightarrow\left(x^{*}, 0\right)$ and $\left\{\delta_{k}\right\} \rightarrow 0$, the set of $\delta_{k}$-active constraints of $(2)$ at $\left(x^{k}, \zeta_{k}\right)$ is a subset of the active constraints at $\left(x^{*}, 0\right)$ for all $k \in \mathcal{S}$ sufficiently large. Then, defining $d^{k}$ by $(29)$, the proof of Theorem 4 can be readily adapted to show that there is a threshold $c^{*}$ (depending on $x^{*}$ and $C$ from Definition 7 ) such that

$$
\left(G_{i}^{T} x^{k}\right)\left(H_{i}^{T} x^{k}\right)=0, \quad i \in I_{G} \cap I_{H},
$$

for all $k \in \mathcal{S}$ sufficiently large with $c_{k} \geq c^{*}$.

Suppose that $\tau_{i}^{*}<0$ for some $i \in I_{G} \cap I_{H}$. Since $\tau_{i}^{k} \geq 0$ and (23c) holds when restricted to $k \in \mathcal{S}$, we have

$$
c_{k} H_{i}^{T} x^{k}=\tau_{i}^{k}-\tau_{i}^{*}+o(1) \geq-\tau_{i}^{*} / 2,
$$

for all $k \in \mathcal{S}$ sufficiently large. This inequality implies that $H_{i}^{T} x^{k}>0$ and so, by (30), $G_{i}^{T} x^{k}=0$. Since (23d) holds when restricted to $k \in \mathcal{S}$, this in turn implies

$$
\nu_{i}^{k}-c_{k} G_{i}^{T} x^{k}=\nu_{i}^{k} \rightarrow \nu_{i}^{*}, \text { as } k \in \mathcal{S}, k \rightarrow \infty .
$$

From (31) and the eighth row of (10), we have

$$
\nu_{i}^{k} \leq \frac{\epsilon_{k}}{H_{i}^{T} x^{k}} \leq \frac{2 \epsilon_{k} c_{k}}{-\tau_{i}^{*}} \rightarrow 0, \quad \text { as } k \in \mathcal{S}, k \rightarrow \infty,
$$

so we conclude that $\nu_{i}^{*}=0$. Similar reasoning shows that if $\nu_{i}^{*}<0$ for some $i \in I_{G} \cap I_{H}$, then $\tau_{i}^{*}=0$. Hence, the accumulation point $x^{*}$ is M-stationary.

(b) Suppose that $\left\{c_{k}\right\}$ is bounded by a constant $\bar{c}$. We argue by contradiction. Suppose that $\tau_{i}^{*}<0$ for some $i \in I_{G} \cap I_{H}$. We have from (31) that

$$
H_{i}^{T} x^{k} \geq \frac{-\tau_{i}^{*}}{2 c_{k}} \geq \frac{-\tau_{i}^{*}}{2 \bar{c}},
$$

for all $k \in \mathcal{S}$ sufficiently large. This bound contradicts $\left\{H_{i}^{T} x^{k}\right\}_{k \in \mathcal{S}} \rightarrow 0$. Similar logic shows that we cannot have $\nu_{i}^{*}<0$ for any $i \in I_{G} \cap I_{H}$. Hence $\tau_{i}^{*} \geq 0$ and $\nu_{i}^{*} \geq 0$ for all $i \in I_{G} \cap I_{H}$, so $x^{*}$ is strongly stationary.

(c) For any $i \in I_{G} \backslash I_{H}$, we have as in the final part of the proof of Theorem 4 that $\tau_{i}^{k}>0$ for all $k \in \mathcal{S}$ sufficiently large. It follows from exact complementarity that $G_{i}^{T} x^{k}=0$ for all $k$ sufficiently large. An analogous argument shows that $H_{i}^{T} x^{k}=0$ for all $i \in I_{H} \backslash I_{G}$ and all $k$ sufficiently large. These observations 
together with (30) yield that $\left(G_{i}^{T} x^{k}\right)\left(H_{i}^{T} x^{k}\right)=0$ for all $i=1,2, \ldots, m$ and all $k$ sufficiently large with $c_{k} \geq c^{*}$, thus proving the result.

By Lemma 1, the feasibility of $x^{*}$ is guaranteed under Assumption 1. Thus, if we further assume in Theorem 5 that $\left\{\left(x^{k}, \zeta_{k}\right)\right\}$ satisfies Assumption 1, then the assumption that $x^{*}$ is feasible for (1) can be dropped.

\subsection{Another Condition for Finite Exact Complementarity}

In this subsection, we describe a strengthened version of the MPEC-LICQ under which exact complementarity $\left(G^{T} x^{k}\right)^{T}\left(H^{T} x^{k}\right)=0$ holds for sufficiently large $c_{k}$, where $\left(x^{k}, \zeta_{k}\right)$ is an inexact second-order point of $\operatorname{PF}\left(c_{k}\right)$. In contrast to Theorem 5(c), exact complementarity between bound constraints and their multipliers need not hold at all iterations.

Definition 8. The strengthened MPEC-LICQ (MPEC-SLICQ) holds at a feasible point $x^{*}$ of (1) if the vectors in each of the following sets are linearly independent:

$$
\mathcal{K} \cup\left\{H_{j}\right\}, \text { for } j \in I_{G} \backslash I_{H}, \quad \mathcal{K} \cup\left\{G_{j}\right\}, \text { for } j \in I_{H} \backslash I_{G},
$$

where $\mathcal{K}$ is the set of active constraint gradients at $x^{*}$ defined in (7).

Clearly, MPEC-SLICQ $\Rightarrow$ MPEC-LICQ. Using this constraint qualification, we have the following result.

Theorem 6. Let $\left\{c_{k}\right\},\left\{\epsilon_{k}\right\}$, and $\left\{\delta_{k}\right\}$ be nonnegative sequences with $c_{k}>0$ nondecreasing with $k,\left\{c_{k} \epsilon_{k}\right\} \rightarrow 0$, and $\left\{\delta_{k}\right\} \rightarrow 0$. Suppose that $\left(x^{k}, \zeta_{k}\right)$ is an $\left(\epsilon_{k}, \delta_{k}\right)$-second-order point of $P F\left(c_{k}\right), k=0,1, \ldots$ Let $\mathcal{S} \subset\{0,1, \ldots\}$ be such that $\left\{x^{k}\right\}_{k \in \mathcal{S}} \rightarrow x^{*}$, where $x^{*}$ is feasible for (1) and satisfies MPEC-SLICQ. Then there is a threshold $c^{*}$ such that, for all $k \in \mathcal{S}$ sufficiently large with $c_{k} \geq c^{*}$, we have $\left(G^{T} x^{k}\right)^{T}\left(H^{T} x^{k}\right)=0$.

Proof. We have from the proof of Theorem 4(a) that (30) holds, that is, $\left(G_{j}^{T} x^{k}\right)\left(H_{j}^{T} x^{k}\right)=0$ for all $j \in I_{G} \cap I_{H}$ and all $k \in \mathcal{S}$ sufficiently large so that $c_{k} \geq c^{*}$, for a suitably large $c^{*}$.

For $j \in I_{G} \backslash I_{H}$, suppose for contradiction that $G_{j}^{T} x^{k}>0$ for infinitely many $k \in \mathcal{S}$. Since $\left\{x^{k}\right\}_{k \in \mathcal{S}} \rightarrow x^{*}$, we have $H_{j}^{T} x^{k}>0$ for all $k \in \mathcal{S}$ sufficiently large. Define a direction $d^{k}$ similarly to (29) as follows:

$$
\begin{aligned}
\nabla g_{i}\left(x^{k}\right)^{T} d^{k} & =0, \quad \text { for } i \in I_{g}, \\
\nabla h\left(x^{k}\right)^{T} d^{k} & =0, \quad \text { for } i \in I_{G} \text { with } i \neq j, \\
G_{i}^{T} d^{k} & =0, \quad \text { for } i \in I_{H}, \\
H_{i}^{T} d^{k} & =0, \quad \\
G_{j}^{T} d^{k} & =1, \\
H_{j}^{T} d^{k} & =-1 .
\end{aligned}
$$

Since MPEC-SLICQ holds at $x^{*}$, the gradients in this definition are approaching the linearly independent set $\mathcal{K} \cup\left\{H_{j}\right\}$, so $d^{k}$ satisfying (32) exists for all $k \in \mathcal{S}$ 
sufficiently large and can be chosen so that $\left\|d^{k}\right\|=O(1)$. Moreover, for $k \in \mathcal{S}$ sufficiently large, $\left(d^{k}, 0\right)$ lies in the direction set defined in Definition 7 . We can now follow the proof of Theorem 4 to obtain a contradiction. Thus $G_{j}^{T} x^{k}=0$ for all $k \in \mathcal{S}$ sufficiently large.

A similar argument shows that $H_{j}^{T} x^{k}=0$ for all $j \in I_{H} \backslash I_{G}$, and all $k \in \mathcal{S}$ sufficiently large. The result then follows. $\square$

By Lemma 1, if we further assume in Theorem 6 that $\left\{\left(x^{k}, \zeta_{k}\right)\right\}$ satisfies Assumption 1, then the assumption that $x^{*}$ is feasible for (1) can be dropped.

A natural question to ask is whether MPEC-SLICQ has a high likelihood of holding at the solution point. We approach this question experimentally, in the numerical examples that we discuss in Section 5. We leave as an open question whether there is some quantifiable way to assert that MPEC-SLICQ holds "almost always" or "generically." That is, can we define a function space containing the problem data, and a topology in the space, and then argue that the property in question holds for an open and dense subset in the data space? In this vein, Jongen, Jonker, and Twilt $[10,11]$ have shown that LICQ holds "almost always" for nonlinear programs, and Scholtes and Stöhr [24] have shown that MPEC-LICQ holds "almost always" for MPEC.

\subsection{An Algorithm Based on Inexact Second-Order Points}

In this subsection, we analyze a practical algorithm that generates a sequence of inexact second-order points $\left(x^{k}, \zeta_{k}\right)$ of $\mathrm{PF}\left(c_{k}\right)$. This algorithm will be the basis of our computational study in Section 5.

\section{Algorithm Elastic-Inexact}

Choose parameters $c_{0}>0, \epsilon_{0}>0, M_{\epsilon}>M_{c}>1$, and positive sequences

$$
\left\{\delta_{k}\right\} \rightarrow 0,\left\{\omega_{k}\right\} \rightarrow 0
$$

for $k=0,1,2, \ldots$

find an $\left(\epsilon_{k}, \delta_{k}\right)$-second-order point $\left(x^{k}, \zeta_{k}\right)$ of $\mathrm{PF}\left(c_{k}\right)$ with

Lagrange multipliers $\left(\lambda^{k}, \mu^{-k}, \mu^{+k}, \tau^{k}, \nu^{k}, \pi^{-k}, \pi^{+k}\right)$;

if $\zeta_{k}+\left(G^{T} x^{k}\right)^{T}\left(H^{T} x^{k}\right) \geq \omega_{k}$,

set $c_{k+1}=M_{c} c_{k}$

else

end (if)

$$
\text { set } c_{k+1}=c_{k}
$$

choose $\epsilon_{k+1} \in\left(0, \epsilon_{k} / M_{\epsilon}\right]$.

\section{end (for)}

Notice that our algorithmic choices imply that, for all $k$,

$$
\frac{c_{k+1} \epsilon_{k+1}}{c_{k} \epsilon_{k}} \leq \frac{M_{c}}{M_{\epsilon}}<1
$$

We show below that the sequences generated by Algorithm Elastic-Inexact satisfy Assumption 1(c), and we accordingly apply Lemma 1 and Theorems 5 and 6 to obtain the desired convergence result. 
Theorem 7. Consider the sequences generated by Algorithm Elastic-Inexact. Suppose that they satisfy Assumption 1(a),(b). Then every accumulation point $x^{*}$ of $\left\{x^{k}\right\}$ is feasible for (1). If $x^{*}$ satisfies MPEC-LICQ, then the following results hold.

(a) $x^{*}$ is M-stationary for (1).

(b) Suppose that $\left\{c_{k}\right\}$ is bounded. Then $x^{*}$ is strongly stationary for (1).

(c) Suppose that either $x^{*}$ satisfies MPEC-SLICQ or $\tau^{k} \perp G^{T} x^{k}$ and $\nu^{k} \perp H^{T} x^{k}$ for all $k$. Let $\mathcal{S} \subset\{0,1, \ldots\}$ be such that $\left\{x^{k}\right\}_{k \in \mathcal{S}} \rightarrow x^{*}$. Then, there is a threshold $c^{*}$ such that, for all $k \in \mathcal{S}$ sufficiently large with $c_{k} \geq c^{*}$, we have $\left(G^{T} x^{k}\right)^{T}\left(H^{T} x^{k}\right)=0$.

Proof. Clearly $\left\{c_{k}\right\}$ is nondecreasing, and, by (33), $\left\{c_{k} \epsilon_{k}\right\} \rightarrow 0$. Also, since $c_{k+1}=M_{c} c_{k}$ whenever $\zeta_{k}+\left(G^{T} x^{k}\right)^{T}\left(H^{T} x^{k}\right) \geq \omega_{k}$, we see that Assumption 1 (c) is satisfied. (Take $\eta_{k}=c_{k}$ if $k \leq \bar{k}$, and take $\eta_{k}=c_{k}+k$ if $k>\bar{k}$, where $\bar{k} \stackrel{\text { def }}{=} \sup \left\{k \mid \zeta_{k}+\left(G^{T} x^{k}\right)^{T}\left(H^{T} x^{k}\right) \geq \omega_{k}\right\}$.) Then, by Lemma 1, every accumulation point $x^{*}$ of $\left\{x^{k}\right\}$ is feasible for (1). This observation, in conjunction with Theorems 5 and 6 , yields the desired conclusion.

An interesting research topic is to identify the conditions under which $\left\{c_{k}\right\}$ remains bounded in Algorithm Elastic-Inexact.

\section{Discussion and Examples}

In this section, we present two examples that illustrate the convergence behaviors (both strengths and limitations) of the algorithms studied in Section 3. We also compare them with the convergence behaviors of the regularized formulation of Scholtes [23] on these examples, illustrating why global convergence results similar to those in Section 3 cannot be proved for the latter formulation.

\subsection{Regularized Formulation}

In Scholtes [23], the problem (1) is approximated by the following nonlinear program:

$$
\begin{array}{rl}
\operatorname{Reg}(t): \quad \min _{x} & f(x) \quad \text { subject to } \\
& g(x) \geq 0, \quad h(x)=0, \\
& G^{T} x \geq 0, \quad H^{T} x \geq 0, \quad\left(G_{i}^{T} x\right)\left(H_{i}^{T} x\right) \leq t, \quad i=1,2, \ldots, m,
\end{array}
$$

where $t$ is a nonnegative parameter. For $t>0$, this formulation tends to avoid the constraint degeneracy associated with the nonlinear programming formulation of (1). Scholtes [23, Theorem 4.1] showed that if (1) has a strongly stationary point $x^{*}$ that satisfies MPEC-LICQ and certain second-order sufficient optimality conditions, then for $t>0$ sufficiently small, $\operatorname{Reg}(t)$ has a first-order point $x(t)$ near $x^{*}$ that satisfies LICQ and second-order sufficient optimality conditions for (34). Generally, however, $x(t)$ does not coincide with $x^{*}$ for $t>0$ sufficiently small 
(unless restrictive assumptions are made). The relationship between $\operatorname{Reg}(t)$ and (1) is studied also by Ralph and Wright [20].

The following theorem summarizes global convergence properties for algorithms based on exact solutions of the regularized formulation.

Theorem 8. Let $\left\{t_{k}\right\}$ be a sequence of positive scalars decreasing to 0 , and let $x^{k}$ be a first-order point of Reg $\left(t_{k}\right)$ with $\left\{x^{k}\right\} \rightarrow x^{*}$, where MPEC-LICQ holds at $x^{*}$. Then $x^{*}$ is C-stationary for (1).

If, in addition, $x^{k}$ is a second-order point of Reg(t $\left.t_{k}\right)$ (defined analogously as for $\left.P F\left(c_{k}\right)\right)$ for all $k$, then $x^{*}$ is M-stationary for (1).

Proof. See Scholtes [23, Theorems 3.1 and 3.3].

It also follows from the proof of [23, Theorem 3.3] that if, in addition, each $\left(G_{i}^{T} x^{k}\right) /\left(H_{i}^{T} x^{k}\right), i=1,2, \ldots, m$ is uniformly bounded between two positive scalars (akin to the asymptotically weakly nondegeneracy condition of Fukushima and Pang [6]), then $x^{*}$ is strongly stationary.

Scholtes showed that Theorem 8 remains true when the last $m$ constraints in (34) are replaced by a single constraint $\left(G^{T} x\right)^{T}\left(H^{T} x\right) \leq t$ [23, Appendix]. In fact, any first-order point of this variant of $\operatorname{Reg}(t)$ is a first-order point of the variant of $\mathrm{PF}(c)$ with $\zeta$ fixed at zero, where $c$ is the multiplier for the constraint $\left(G^{T} x\right)^{T}\left(H^{T} x\right) \leq t$. This suggests that the first-order points of $\operatorname{Reg}\left(t_{k}\right)$ and $\mathrm{PF}\left(c_{k}\right)$ have similar asymptotic properties, which is corroborated by Theorems 3 and 8.

A natural question is whether a finite termination property like those in Section 3 that are based on second-order points of $\mathrm{PF}\left(c_{k}\right)$ also holds for secondorder points of $\operatorname{Reg}\left(t_{k}\right)$. Example 2 below answers this question in the negative. We show in this example that a sequence of second-order points of $\operatorname{Reg}\left(t_{k}\right)$ may approach an M-stationary point of (1) that is not strongly stationary.

\subsection{Examples}

Our first example is one in which the algorithms of Section 3 can terminate at local solutions of (1), while algorithms based on the regularization $\operatorname{Reg}\left(t_{k}\right)$ achieve convergence to the global solution of (1).

Example 1. Consider the following MPEC in two variables:

$$
\min _{x} \frac{1}{2}\left(x_{2}-1\right)^{2} \quad \text { subject to } 0 \leq x_{1} \perp x_{2} \geq 0,
$$

whose global solution is $x=(0,1)$ but which also has strongly stationary points at $\left(x_{1}, 0\right)$ for all $x_{1}>0$. $((0,0)$ is only M-stationary.) The elastic-mode formulation is

$$
\operatorname{PF}\left(c_{k}\right): \quad \min _{x} \frac{1}{2}\left(x_{2}-1\right)^{2}+c_{k} x_{1} x_{2} \quad \text { subject to } x_{1} \geq 0, x_{2} \geq 0,
$$

with $c_{k}>0$. The Hessian of the Lagrangian function for $\operatorname{PF}\left(c_{k}\right)$ is

$$
\nabla_{x x}^{2} L_{c_{k}}(x, \tau, \nu)=\left[\begin{array}{cc}
0 & c_{k} \\
c_{k} & 1
\end{array}\right]
$$


respectively. Since the Hessian has a negative eigenvalue for all $c_{k}>0$, it follows that at any second-order point of (36), at least one of the bounds $x_{1} \geq 0$ or $x_{2} \geq 0$ must be active. Hence, such a point is feasible for (35), and Algorithm ElasticExact terminates finitely, as indicated in Corollary 2. One such second-order point is $x^{k}=(0,1)$, which is a global solution of (35) satisfying MPEC-LICQ. The other second-order points of (36) are

$$
x^{k}(\alpha)=\left[\begin{array}{c}
\frac{1}{c_{k}}+\alpha \\
0
\end{array}\right], \quad \text { for all } \alpha \geq 0,
$$

which are all local solutions of (35) satisfying MPEC-LICQ. Algorithm ElasticExact may terminate finitely at one of these local solutions.

The regularized formulation of (35) is

$$
\operatorname{Reg}\left(t_{k}\right): \quad \min _{x} \frac{1}{2}\left(x_{2}-1\right)^{2} \quad \text { subject to } x_{1} \geq 0, x_{2} \geq 0, x_{1} x_{2} \leq t_{k} .
$$

It can be verified that, for each $t_{k}>0$, the first-order points of (37) are

$$
x^{k}(\alpha)=\left[\begin{array}{c}
\alpha \\
1
\end{array}\right], \quad \alpha \in\left[0, t_{k}\right],
$$

all of which are global solutions and second-order points of (37). In particular, any sequence of first-order points of $\operatorname{Reg}\left(t_{k}\right)$ will converge to the global solution of (35).

The behavior of Algorithm Elastic-Exact in Example 1 above is not particularly surprising. If it terminates finitely at a point $\left(x_{1}, 0\right)$ with $x_{1}>0$, it yields a local solution of (1), which is all we expect from most optimization methods. For large $c_{k}$, however, points with lesser function values are "just around the corner" from these local solutions. Algorithms that can detect such local solutions reliably and move away from them along feasible descent directions are the subject of ongoing investigation.

The next example demonstrates that a sequence of second-order points of $\operatorname{Reg}\left(t_{k}\right)$ may approach an M-stationary point of (1) while Algorithm ElasticExact terminates finitely at a strongly stationary point of (1). It is constructed from Robinson's [21] example of a strict local solution that is not isolated.

Example 2. Consider the function $F: \mathbb{R} \rightarrow \mathbb{R}$ defined as follows:

$$
F(y) \stackrel{\text { def }}{=} \int_{0}^{y} t^{6} \sin (1 / t) d t .
$$

It is easy to verify that $F$ is three times continuously differentiable over $\mathbb{R}$. Our example MPEC is

$$
\min _{x}-x_{1}-F\left(x_{2}\right) \quad \text { subject to } \quad 0 \leq x_{1} \perp x_{2} \geq 0,
$$

where $x_{1}$ and $x_{2}$ are scalars. It is not difficult to verify that this MPEC has an M-stationary point at $x^{*}=(0,0)$, with unique multipliers $\tau^{*}=-1$ and 
$\nu^{*}=0$, and that the MPEC-LICQ is satisfied at this point. It also has strongly stationary points arbitrarily near $(0,0)$, as we shall see.

We consider the behavior of Algorithm Elastic-Exact on this MPEC and then show how the results of Section 3 apply. The elastic-mode formulation of (38) is

$$
\mathrm{PF}(c): \min _{x}-x_{1}-F\left(x_{2}\right)+c x_{1} x_{2} \quad \text { subject to } x_{1} \geq 0, x_{2} \geq 0,
$$

with $c>0$. Its first-order stationarity conditions are

$$
0 \leq\left[\begin{array}{c}
-1+c x_{2} \\
-F^{\prime}\left(x_{2}\right)+c x_{1}
\end{array}\right] \perp\left[\begin{array}{l}
x_{1} \\
x_{2}
\end{array}\right] \geq 0 .
$$

The Hessian of the Lagrangian for (39) is

$$
\nabla_{x x}^{2} L_{c}(x, \tau, \nu)=\left[\begin{array}{cc}
0 & c \\
c-F^{\prime \prime}\left(x_{2}\right)
\end{array}\right] .
$$

Since this matrix has a negative eigenvalue for all $c>0$, it follows that at any second-order point of (39), at least one of the bounds $x_{1} \geq 0$ or $x_{2} \geq 0$ must be active. Hence, such a point is feasible for (38), and Algorithm Elastic-Exact terminates finitely, as indicated in Corollary 2.

More precisely, notice that the second-order points of (39) cannot have the form $\left(x_{1}, 0\right)$ for any $x_{1} \geq 0$, as the first-order conditions (40) cannot be satisfied at such points. Hence, the second-order points of $\mathrm{PF}(c)$ must be $\left(0, x_{2}\right)$ for some $x_{2}>0$. From (40) and the second-order necessary optimality conditions for (39), we must have

$$
x_{2} \geq \frac{1}{c}, \quad F^{\prime}\left(x_{2}\right)=0, \quad F^{\prime \prime}\left(x_{2}\right) \leq 0,
$$

from which we deduce that

$$
x_{2} \geq \frac{1}{c}, \quad \sin \frac{1}{x_{2}}=0, \quad \cos \frac{1}{x_{2}}=-1,
$$

so that $x_{2}=(2 n \pi+\pi)^{-1}$ for some integer $n \geq 0$. Notice that each of these points is strongly stationary for the MPEC (38).

It is of course possible that the method for solving (39) will avoid these local solutions and detect the direction of unboundedness along the positive $x_{1}$ axis. The outcome that emerges will depend on the particular method and its initialization.

The regularized formulation of (38) is as follows:

$$
\operatorname{Reg}(t): \quad \min _{x}-x_{1}-F\left(x_{2}\right) \quad \text { subject to } x_{1} \geq 0, \quad x_{2} \geq 0, x_{1} x_{2} \leq t .
$$

Its first-order necessary optimality conditions are

$$
\begin{gathered}
{\left[\begin{array}{c}
-1 \\
-F^{\prime}\left(x_{2}\right)
\end{array}\right]-\left[\begin{array}{l}
1 \\
0
\end{array}\right] \tau-\left[\begin{array}{l}
0 \\
1
\end{array}\right] \nu+\left[\begin{array}{l}
x_{2} \\
x_{1}
\end{array}\right] \gamma=\left[\begin{array}{l}
0 \\
0
\end{array}\right],} \\
0 \leq\left[\begin{array}{l}
\tau \\
\nu \\
\gamma
\end{array}\right] \perp\left[\begin{array}{c}
x_{1} \\
x_{2} \\
t-x_{1} x_{2}
\end{array}\right] \geq 0 .
\end{gathered}
$$


Since $\tau \geq 0$, the first equation implies $\gamma \neq 0$ and hence $x_{1} x_{2}=t$ at any firstorder point of (41). This in turn implies $\nu=\tau=0$ and $\gamma=1 / x_{2}$, so that the first-order conditions reduce to

$$
\frac{t}{\left(x_{2}\right)^{2}}-\left(x_{2}\right)^{6} \sin \left(1 / x_{2}\right)=0 .
$$

For certain values of the parameter $t$, it is easy to identify values of $x_{2}$ that satisfy this condition. These include the following $\left(t_{k}, x_{2}^{k}\right)$ pairs, defined for any $k=0,1,2, \ldots$ :

$$
x_{2}^{k}=(2 k \pi+\pi / 6)^{-1}, \quad t_{k}=\frac{1}{2}(2 k \pi+\pi / 6)^{-8}, \quad k=0,1,2, \ldots .
$$

At a first-order point of (41), since the constraint $x_{1} x_{2} \leq t$ is active, a sufficient condition for local optimality is that the Hessian of the Lagrangian be positive definite over the null space of the gradient $\left(x_{2}, x_{1}\right)^{T}$, that is,

$$
\left[\begin{array}{ll}
d_{1} & d_{2}
\end{array}\right]\left[\begin{array}{cc}
0 & \gamma \\
\gamma-F^{\prime \prime}\left(x_{2}\right)
\end{array}\right]\left[\begin{array}{l}
d_{1} \\
d_{2}
\end{array}\right]>0 \text { whenever } x_{2} d_{1}+x_{1} d_{2}=0,\left(d_{1}, d_{2}\right) \neq 0 .
$$

Using $\gamma=1 / x_{2}$ and $x_{1}=t / x_{2}$, one can reduce this condition to the positivity of

$$
\frac{-2 t}{\left(x_{2}\right)^{3}}-6\left(x_{2}\right)^{5} \sin \left(1 / x_{2}\right)+\left(x_{2}\right)^{4} \cos \left(1 / x_{2}\right),
$$

which, when evaluated at the $\left(t_{k}, x_{2}^{k}\right)$ pairs above, yields

$$
-(2 k \pi+\pi / 6)^{-5}-3(2 k \pi+\pi / 6)^{-5}+(\sqrt{3} / 2)(2 k \pi+\pi / 6)^{-4} .
$$

Since the last term dominates for $k$ sufficiently large, the above quantity is positive for all $k$ sufficiently large. It follows that

$$
x^{k}=\left(\frac{t_{k}}{x_{2}^{k}}, x_{2}^{k}\right)=\left(\frac{1}{2}(2 k \pi+\pi / 6)^{-7},(2 k \pi+\pi / 6)^{-1}\right)
$$

is a local minimizer of $\operatorname{Reg}\left(t_{k}\right)$ satisfying second-order sufficient optimality conditions, for all $k$ sufficiently large. Notice that $\left\{x^{k}\right\}$ approaches the M-stationary point $x^{*}=(0,0)$, which is not strongly stationary.

\section{Numerical Results}

We present numerical results for Algorithm Elastic-Inexact applied to a set of 18 MPECs, showing the outcomes for each problem and analyzing whether exact complementarity, MPEC-LICQ, MPEC-SLICQ, or the second-order conditions were satisfied at the final point generated by the algorithm. 


\subsection{Problems and Formulation Details}

Our MPECs all arise from a model of an elastic membrane hanging on top of an obstacle. The membrane is attached to a support whose shape is parametrized by the variables in the optimization problem. Descriptions of all our problems can be found in Outrata, Kocvara, and Zowe [18]. We use the AMPL formulations from the MacMPEC Library of Leyffer [13]; see also Fletcher and Leyffer [5].

- Incidence set identification [18, Section 9.4]. This MPEC seeks the shape of the support so that the contact region is as close as possible to a prescribed shape. The objective function is a measure of the difference between the current and desired shapes of the contact region. This problem class is referred to as incid-set by [5]; we use the abbreviation is.

- Packaging problem with pliant obstacle [18, Section 9.3]. This MPEC seeks the shape of the support that minimizes the area of the membrane while keeping the membrane in contact with the obstacle over at least a prescribed region. The obstacle here is pliant - it can deform under pressure from the membrane - while the objective function is the area of the membrane. This class is referred to as pack-comp by [5]; we use the abbreviation pc.

- Packaging problem with rigid obstacle [18, Section 9.2]. This MPEC is the same as the previous one except that now the obstacle is rigid. This class is referred to as pack-rig by [5]; we use the abbreviation pr.

We formulate six variants of each of these problems. First, we perform finite element discretizations on three different grids. We include the strings 8,16 , and 32 in the problem names to indicate $8 \times 8,16 \times 16$, and $32 \times 32$ grids, respectively. Second, we consider two types of obstacles. A linear obstacle [18, Example 9.1] is indicated by the digit 1 in the problem name, while a parabolic obstacle [18, Example 9.2] is indicated by the digit 2 .

We implemented Algorithm Elastic-Inexact with parameters $c_{0}=10, \epsilon_{0}=$ $10^{-3}, M_{\epsilon}=15, M_{c}=10, \omega_{k}=\min \left\{(k+1)^{-1}, c_{k}^{-1 / 2}\right\}$. To solve each subproblem $\operatorname{PF}\left(c_{k}\right)$, we used the filterSQP code of Fletcher and Leyffer [5]. This code uses active set and second-order information and thus is likely to produce inexact solutions that satisfy the assumptions of Theorem 7. (The parameter $\delta_{k}$, which determines approximate activeness of the constraints, is determined internally by filterSQP.) The algorithm is terminated at $\left(x^{k}, \zeta_{k}\right)$ when either $\zeta_{k}+\left(G^{T} x^{k}\right)^{T}\left(H^{T} x^{k}\right) \leq 10^{-7}$ and $\epsilon_{k} \leq 10^{-3} 15^{-6}$ (at least 6 tolerance updates) or filterSQP is unable to solve the subproblem $\mathrm{PF}\left(c_{k}\right)$ to optimality at some iteration $k$, where $\epsilon_{k} \geq 10^{-3} 15^{-8}$ (that is, before 8 tolerance updates have been made).

Before describing the results, we give a few more details of our AMPL formulations. First, we note that the models contain a discretization of a state variable and of a differential operator. The equality constraints that do not include the action of the operator are not relaxed. For each of the equality constraints that include the action (those arising from the Galerkin equations for the finite elements), we replaced its zero right-hand side by a slack variable and constrained the slack to lie between $-\zeta$ and $\zeta$, as in the formulation (2). Our reasons for 
formulating the equality constraints in this way have to do with the postprocessing required to compute the diagnostic quantities for our results. The theory of earlier sections still applies.

The constraints $\zeta \geq 0, G^{T} x \geq 0$, and $H^{T} x \geq 0$ were implemented as bound constraints, defined at the time the corresponding variables were declared. The upper bound constraint $\zeta \leq \bar{\zeta}$ was omitted because $\left\{\zeta_{k}\right\}$ remained bounded without it. Preprocessing was turned off, as AMPL tends to "process out" the bound constraints when it is enabled.

Bound constraints are also present on some of the (noncomplementary) variables in the models. When defined as bounds in AMPL, these are treated differently from general inequalities. For purposes of our tests, we define the set of components of the final point $x^{k}$ that are away from their respective bounds as follows:

$$
\mathcal{F}=\left\{j \in\{1,2, \ldots, n\} \mid l_{j}+\delta \leq x_{j}^{k} \leq u_{j}-\delta\right\},
$$

where $l$ and $u$ are the vectors of lower and upper bounds on $x$ and $\delta$ is a tolerance (we used $\delta=10^{-6}$ ). In forming the Jacobian and Hessian matrices, we retain only the rows and columns corresponding to "free" variables - those indexed by $\mathcal{F}$. We use $n_{F}$ to denote the number of elements in $\mathcal{F}$.

Checking of the MPEC-LICQ condition at the final point $x^{k}$ requires us to collect the gradients of the equality and inequality constraint functions at $x^{k}$, along with the active constraint indices for the inequality constraints and the bound constraints $G^{T} x \geq 0$ and $H^{T} x \geq 0$. Postprocessing is required to extract this information, to remove the "duplication" of the equality constraints that occurs as a result of the relaxation of these constraints. As AMPL provides no simple mechanism to do this, we wrote the final gradients into a file and processed it with Perl and shell scripts to remove duplicates and generate the active constraint gradients.

\subsection{Quality of Computed Solutions}

Feasibility and optimality properties of the final point $x^{k}$ generated by our algorithm are shown in Table 1 . We show the final objective function value $(\mathrm{Obj})$, the manner in which filterSQP terminated on solving the last subproblem, the final values of the algorithmic parameters $c_{k}$ and $\epsilon_{k}$, and the final value of the infeasibility (violation of the MPEC constraints) at $x^{k}$.

As a measure of the complementarity at the final point we define the indicator

$$
\text { CompInd }=\max _{i=1,2, \ldots, m} \min \left\{G_{i}^{T} x^{k}, H_{i}^{T} x^{k}\right\} .
$$

On all problems, a final point with $\zeta_{k}=0$ and CompInd $=0$ was obtained. Feasibility was attained to high precision on all problems but is-1-32. The code filterSQP terminated normally on all but three of the problems. On two problems, an error message indicated difficulty in finding a feasible point locally in 1,000 or fewer iterations ("Local Inf"). On the remaining problem, the trust 


\begin{tabular}{|l|rl|lll|}
\hline Problem & Obj & Termination Message & $c_{k}$ & $\epsilon_{k}$ & Infeas \\
\hline is-1-8 & $3.81 \mathrm{e}-17$ & Optimal & 10 & $10^{-3} 15^{-6}$ & $2.60 \mathrm{e}-17$ \\
is-1-16 & $1.20 \mathrm{e}-16$ & Optimal & 10 & $10^{-3} 15^{-6}$ & $3.14 \mathrm{e}-13$ \\
is-1-32 & $2.33 \mathrm{e}-07$ & Small Trust Region & 10 & $10^{-3} 15^{-6}$ & $2.25 \mathrm{e}-07$ \\
is-2-8 & $5.04 \mathrm{e}-03$ & Optimal & 10 & $10^{-3} 15^{-6}$ & $6.20 \mathrm{e}-17$ \\
is-2-16 & $2.99 \mathrm{e}-03$ & Optimal & 10 & $10^{-3} 15^{-6}$ & $1.93 \mathrm{e}-15$ \\
is-2-32 & $1.77 \mathrm{e}-03$ & Optimal & $10^{3}$ & $10^{-3} 15^{-7}$ & $1.86 \mathrm{e}-15$ \\
\hline pc-1-8 & $6.00 \mathrm{e}-01$ & Optimal & 10 & $10^{-3} 15^{-6}$ & $5.89 \mathrm{e}-17$ \\
pc-1-16 & $6.16 \mathrm{e}-01$ & Optimal & $10^{2}$ & $10^{-3} 15^{-6}$ & $8.26 \mathrm{e}-14$ \\
pc-1-32 & $6.52 \mathrm{e}-01$ & Optimal & $10^{3}$ & $10^{-3} 15^{-6}$ & $3.49 \mathrm{e}-17$ \\
pc-2-8 & $6.73 \mathrm{e}-01$ & Optimal & $10^{2}$ & $10^{-3} 15^{-6}$ & $3.81 \mathrm{e}-17$ \\
pc-2-16 & $7.27 \mathrm{e}-01$ & Optimal & $10^{5}$ & $10^{-3} 15^{-7}$ & $1.10 \mathrm{e}-13$ \\
pc-2-32 & $7.82 \mathrm{e}-01$ & Local Inf & $10^{4}$ & $10^{-3} 15^{-6}$ & $6.06 \mathrm{e}-12$ \\
\hline pr-1-8 & $7.87 \mathrm{e}-01$ & Optimal & $10^{2}$ & $10^{-3} 15^{-6}$ & $2.77 \mathrm{e}-17$ \\
pr-1-16 & $8.26 \mathrm{e}-01$ & Optimal & $10^{3}$ & $10^{-3} 15^{-6}$ & $4.38 \mathrm{e}-13$ \\
pr-1-32 & $8.50 \mathrm{e}-01$ & Optimal & $10^{6}$ & $10^{-3} 15^{-8}$ & $3.31 \mathrm{e}-15$ \\
pr-2-8 & $7.80 \mathrm{e}-01$ & Optimal & $10^{2}$ & $10^{-3} 15^{-6}$ & $1.21 \mathrm{e}-16$ \\
pr-2-16 & $1.08 \mathrm{e}+00$ & Optimal & $10^{5}$ & $10^{-3} 15^{-6}$ & $1.07 \mathrm{e}-16$ \\
pr-2-32 & $1.13 \mathrm{e}+00$ & Local Inf & $10^{6}$ & $10^{-3} 15^{-8}$ & $5.68 \mathrm{e}-13$ \\
\hline
\end{tabular}

Table 1. Numerical Results: Final Objective Function, Termination Message from filterSQP, Final Parameters and Constraint Infeasibility.

region became too small ("Small Trust Region"). We interpret abnormal termination of filterSQP as an indication that the solver could not find an inexact first-order point of the last subproblem.

We note that because CompInd is exactly zero for each problem (even those for which abnormal termination was reported), the outcome of Theorem 7 (c) is verified. Computation of CompInd requires only comparisons (not floatingpoint arithmetic operations); hence, we can attain CompInd $=0$, even in finiteprecision arithmetic.

\subsection{Numerical Verification of Constraint Qualifications}

We now investigate numerically whether the constraint qualifications assumed at various points in this paper are indeed satisfied. We include in our investigation even the three problems with abnormal termination, because the final points generated for those problems are almost feasible and satisfy exact complementarity.

We used the interface to Matlab that is provided by AMPL to import from filterSQP the primal variables $\left(x^{k}, \zeta_{k}\right)$, the Lagrange multipliers

$\left(\lambda^{k}, \mu^{-k}, \mu^{+k}, \tau^{k}, \nu^{k}\right)$, the constraint gradients, and the Hessian of the Lagrangian for the last subproblem $\mathrm{PF}\left(c_{k}\right)$. (See Gay [8] for details on access to AMPL structures by functions defined in Matlab.) We then compute the active sets at $x^{k}$, using a tolerance of $\delta=10^{-6}$, as follows:

$$
\begin{gathered}
I_{g} \stackrel{\text { def }}{=}\left\{i \in\{1,2, \ldots, p\} \mid g_{i}\left(x^{k}\right) \leq \delta\right\}, \\
I_{G} \stackrel{\text { def }}{=}\left\{i \in\{1,2, \ldots, m\} \mid G_{i}^{T} x^{k} \leq \delta\right\}, \\
I_{H} \stackrel{\text { def }}{=}\left\{i \in\{1,2, \ldots, m\} \mid H_{i}^{T} x^{k} \leq \delta\right\} .
\end{gathered}
$$


With these definitions, we compute the gradients of the active constraints, and extract those components that correspond to free variables $\mathcal{F}$. Our reduced Jacobian is therefore defined as follows:

$$
J_{\text {act }} \stackrel{\text { def }}{=}\left[\left[\nabla g_{i}\left(x^{k}\right)\right]_{i \in I_{g}},\left[\nabla h_{i}\left(x^{k}\right)\right]_{i=1,2, \ldots, q},\left[G_{i}\right]_{i \in I_{G}},\left[H_{i}\right]_{i \in I_{H}}\right]_{\mathcal{F}} .
$$

(The final subscript $\mathcal{F}$ indicates that we save only the free-variable rows.) We also define the Hessian of the Lagrangian with respect to the free components of $x$ as follows:

$$
H \stackrel{\text { def }}{=}\left[\frac{\partial^{2}}{\partial x_{i} \partial x_{j}} L_{c_{k}}\left(x^{k}, \zeta_{k}, \lambda^{k}, \mu^{-k}, \mu^{+k}, \tau^{k}, \nu^{k}\right)\right]_{i \in \mathcal{F}, j \in \mathcal{F}} .
$$

We compute the following QR decomposition of $J_{\text {act }}$ :

$$
J_{\text {act }} \Pi=Q\left[\begin{array}{c}
R \\
0
\end{array}\right],
$$

where $\Pi$ is an $m_{\text {act }} \times m_{\text {act }}$ permutation matrix, $Q \in \mathbb{R}^{n_{F} \times n_{F}}$ is orthogonal, and $R \in \mathbb{R}^{m_{\text {act }} \times m_{\text {act }}}$ is upper triangular with nonnegative diagonal elements such that $R_{11} \geq R_{22} \geq \ldots \geq R_{m_{\text {act }}, m_{\text {act }}} \geq 0$.

We use the preceding $\mathrm{Q}$ and $\mathrm{R}$ factors to test satisfaction of the constraint qualification and second-order point assumptions as follows.

- MPEC-LICQ holds (with active set tolerance $\delta$ ) if $R$ is nonsingular. To allow for numerical error, we set $\epsilon_{\text {mach }}$ to IEEE double-precision machine epsilon (approximately $2.2 \times 10^{-16}$; see Overton [19, Table 4.4]) and compute the quantities

$$
\operatorname{cond}_{2}\left(J_{\text {act }}\right) \stackrel{\text { def }}{=} \frac{\sigma_{\max }(R)}{\sigma_{\min }(R)}, \quad \operatorname{cond}_{*}\left(J_{\text {act }}\right) \stackrel{\text { def }}{=} \frac{\max \operatorname{diag}(R)}{\min \operatorname{diag}(R)},
$$

where $\sigma_{\max }$ and $\sigma_{\min }$ denote the smallest and largest singular values, respectively. We judge MPEC-LICQ to be satisfied if

$$
\max \left(\operatorname{cond}_{2}\left(J_{\text {act }}\right), \operatorname{cond}_{*}\left(J_{\text {act }}\right)\right) \text { is "small" compared to } \epsilon_{\text {mach }}^{-1} \text {. }
$$

If MPEC-LICQ holds, we define the matrix $Q_{2}$ to be the last $n_{F}-m_{\text {act }}$ columns of $Q$. The columns of $Q_{2}$ then define an orthonormal basis for the nullspace of $J_{\text {act }}^{T}$. If MPEC-LICQ fails to hold, we define the numerical rank $r$ of $J_{\text {act }}$ to be the largest integer for which $R_{r r} / R_{11}$ is significantly greater than $\epsilon_{\text {mach }}$. In this case, we take $Q_{2}$ to be the last $n_{F}-r$ columns of $Q$. In either case, $Q_{1}$ is the complement of $Q_{2}$ in $Q$.

- MPEC-SLICQ holds if MPEC-LICQ holds and none of the vectors $G_{i}, i \notin I_{G}$ or $H_{i}, i \notin I_{H}$ lie in the span of the columns of $Q_{1}$. Since each column of $\left[\left[G_{i}\right]_{i \in I_{G}},\left[H_{i}\right]_{i \in I_{H}}\right]$ is a column of the identity matrix, and since $Q_{2}$ is the orthogonal complement of $Q_{1}$, a reasonable way to test this condition is to compute the following quantity,

$$
\chi_{\text {span }} \stackrel{\text { def }}{=} \min \left(\min _{i \notin I_{G}}\left\|Q_{2}^{T} G_{i}\right\|_{2}, \min _{i \notin I_{H}}\left\|Q_{2}^{T} H_{i}\right\|_{2}\right),
$$




\begin{tabular}{|c|c|c|c|c|c|c|}
\hline Problem & $n_{F}$ & $m_{\text {act }}$ & $\operatorname{cond}_{2}\left(J_{\text {act }}\right)$ & $\operatorname{cond}_{*}\left(J_{\text {act }}\right)$ & $\chi_{\text {span }}$ & $\lambda_{\min }\left(Q_{2}^{T} H Q_{2}\right)$ \\
\hline is $-1-8$ & 193 & 181 & $3.45 \mathrm{e}+03$ & $3.58 \mathrm{e}+02$ & $1.95 \mathrm{e}-03$ & 0 \\
\hline is $-1-16$ & 763 & 742 & $4.39 \mathrm{e}+04$ & $7.24 \mathrm{e}+03$ & $6.84 \mathrm{e}-04$ & 0 \\
\hline is $-1-32$ & 3042 & 3020 & $5.26 \mathrm{e}+05$ & $8.89 \mathrm{e}+04$ & $3.90 \mathrm{e}-09$ & 0 \\
\hline is $-2-8$ & 184 & 180 & $2.17 \mathrm{e}+03$ & $8.58 \mathrm{e}+01$ & $5.66 \mathrm{e}-04$ & $1.08 \mathrm{e}-04$ \\
\hline is $-2-16$ & 750 & 745 & $6.46 \mathrm{e}+04$ & $1.69 \mathrm{e}+03$ & $8.44 \mathrm{e}-05$ & $4.10 \mathrm{e}-07$ \\
\hline is $-2-32$ & 3032 & 3025 & $\infty$ & $\infty$ & 0 & -1.48 \\
\hline$p c-1-8$ & 228 & 228 & $1.96 \mathrm{e}+02$ & $1.66 \mathrm{e}+01$ & 0 & $\infty$ \\
\hline $\mathrm{pc}-1-16$ & 970 & 964 & $9.38 \mathrm{e}+03$ & $1.75 \mathrm{e}+02$ & $1.91 \mathrm{e}-06$ & $5.55 \mathrm{e}-02$ \\
\hline $\mathrm{pc}-1-32$ & 3997 & 3972 & $4.48 \mathrm{e}+04$ & $2.04 \mathrm{e}+02$ & $1.22 \mathrm{e}-08$ & $4.88 \mathrm{e}-01$ \\
\hline $\mathrm{pc}-2-8$ & 233 & 228 & $3.40 \mathrm{e}+03$ & $1.40 \mathrm{e}+02$ & $1.27 \mathrm{e}-04$ & $1.37 \mathrm{e}+00$ \\
\hline $\mathrm{pc}-2-16$ & 977 & 964 & $1.34 \mathrm{e}+04$ & $1.78 \mathrm{e}+02$ & $4.34 \mathrm{e}-06$ & $6.62 \mathrm{e}-01$ \\
\hline $\mathrm{pc}-2-32$ & 4001 & 3972 & $7.82 \mathrm{e}+04$ & $3.72 \mathrm{e}+02$ & $7.61 \mathrm{e}-09$ & $2.06 \mathrm{e}-01$ \\
\hline pr-1-8 & 186 & 179 & $1.10 \mathrm{e}+03$ & $4.04 \mathrm{e}+02$ & $2.96 \mathrm{e}-17$ & $2.61 \mathrm{e}-07$ \\
\hline $\mathrm{pr}-1-16$ & 754 & 739 & $4.11 \mathrm{e}+03$ & $3.59 \mathrm{e}+02$ & $1.35 \mathrm{e}-18$ & 0 \\
\hline $\mathrm{pr}-1-32$ & 3040 & 3011 & $8.99 \mathrm{e}+07$ & $1.12 \mathrm{e}+06$ & $3.56 \mathrm{e}-19$ & $4.34 \mathrm{e}-01$ \\
\hline pr-2-8 & 185 & 179 & $3.22 \mathrm{e}+03$ & $2.66 \mathrm{e}+01$ & $1.47 \mathrm{e}-18$ & $4.88 \mathrm{e}-01$ \\
\hline $\mathrm{pr}-2-16$ & 743 & 739 & $3.07 \mathrm{e}+03$ & $4.36 \mathrm{e}+01$ & $1.91 \mathrm{e}-23$ & $2.12 \mathrm{e}-01$ \\
\hline pr-2-32 & 3027 & 3011 & $7.62 \mathrm{e}+03$ & $2.63 \mathrm{e}+01$ & $8.92 \mathrm{e}-24$ & $1.79 \mathrm{e}-01$ \\
\hline
\end{tabular}

Table 2. Verification of the Constraint Qualifications and Second-Order Conditions.

and judge MPEC-SLICQ to hold if MPEC-LICQ holds and

$$
\chi_{\text {span }} \text { is "large" compared to } \epsilon_{\text {mach }} \text {. }
$$

- Following Definition 7 , the final primal variables $\left(x^{k}, \zeta_{k}\right)$ form an $\left(\epsilon_{k}, \delta_{k}\right)$ second-order point of $\operatorname{PF}\left(c_{k}\right)$ if they form an $\epsilon_{k}$-first-order point of $\operatorname{PF}\left(c_{k}\right)$ and $u^{T} H u \geq-C\|u\|^{2}$ for all $u \in \mathbb{R}^{n} F$ satisfying $J_{\text {act }}^{T} u=0$, where $C$ is independent of the iteration count. The latter second-order part is equivalent to

$$
\lambda_{\min }\left(Q_{2}^{T} H Q_{2}\right) \geq-C,
$$

where $\lambda_{\min }$ denotes the minimum eigenvalue. Of course, we have no idea how large or how small $C$ should be by looking only at the results from the final iteration. Following the proof of Theorem 4, however, we judge inexact second-order stationarity to hold if

$$
\max \left(0,-\lambda_{\min }\left(Q_{2}^{T} H Q_{2}\right)\right) \text { is "small" compared to "large" } c_{k} \text {. }
$$

The results, tabulated in Table 2, contain in the first three columns the problem name, the number of free variables $n_{F}$, and the number of active constraints $m_{\text {act }}$. Based on these results, we make the following observations.

- We see that $\operatorname{cond}_{2}\left(J_{\text {act }}\right)$ and $\operatorname{cond}_{*}\left(J_{\text {act }}\right)$ are both less than $10^{8} \ll \epsilon_{\text {mach }}^{-1}$ on all problems but is-2-32, where $J_{\text {act }}$ is clearly rank deficient. For these 17 problems we conclude that MPEC-LICQ holds.

- We see that $\chi_{\text {span }}$ is greater than $10^{-9} \gg \epsilon_{\text {mach }}$ on 10 problems, which suggests that MPEC-SLICQ holds for these problems. MPEC-SLICQ appears not to hold on any of the pr problems. It also fails to hold on is-2-32 (as a consequence of failure of MPEC-LICQ) and on pc-1-8 (since $J_{\text {act }}$ is a square nonsingular matrix in this case, so $Q_{2}$ is null and MPEC-SLICQ is trivially not satisfied). 
- We see that $\lambda_{\min }\left(Q_{2}^{T} H Q_{2}\right)$ is nonnegative on all problems on which the matrix is not vacuous, with the single exception of is-2-32, the problem for which MPEC-LICQ failed to hold. If $Q_{2}$ were defined in this case to be the last $n_{F}-m_{\text {act }}$ columns of $Q$, then $Q_{2}^{T} H Q_{2}$ would be positive semidefinite. However, since we define $Q_{2}$ to span the full nullspace of $J_{\text {act }}^{T}$, we obtained the modestly negative number -1.48 shown in the table. Since this number is significantly smaller in absolute value than the final value $10^{3}$ of $c_{k}$, we believe that Definition 7 is satisfied for all 18 problems.

- We note that for all 18 problems the final values of the objective function are very similar to the ones reported in [5].

We conclude that MPECs that satisfy our assumptions are broadly represented in the test problems used in our computational study.

\section{Acknowledgments}

We are grateful to Todd Munson for his advice on AMPL and Matlab and his help with the numerical experiments, and to Sven Leyffer for his help and advice with filterSQP and AMPL. We are also pleased to acknowledge the contributions of two referees, whose close reading and constructive comments led to several improvements. Mihai Anitescu was supported by the Mathematical, Information, and Computational Sciences Division subprogram of the Office of Advanced Scientific Computing Research, Office of Science, U.S. Department of Energy, under Contract W-31-109-ENG-38. Stephen Wright was supported by NSF Grants ATM-0296033, CNS-0127857, CCF-0113051, ANI-0330538, DMS0427689, CCF-0430504, and DOE grant DE-FG02-04ER25627. Paul Tseng was supported by NSF Grant DMS-0511283.

\section{A. A Technical Lemma}

Lemma 2. Let $A$ be a matrix with full column rank and $g$ be a given vector. Let $\left\{A^{k}\right\}$ and $\left\{g^{k}\right\}$ be two sequences such that $\left\{A^{k}\right\} \rightarrow A$ and $\left\{g^{k}\right\} \rightarrow g$, and in addition $g^{k} \in \operatorname{Range}\left(A^{k}\right)$. Then there exist unique $v$ such that $A v=g$ and $a$ sequence $\left\{v^{k}\right\}$, uniquely defined for all $k$ sufficiently large, such that $A^{k} v^{k}=g^{k}$ and $\left\{v^{k}\right\} \rightarrow v$.

Proof. By the given properties, we have $g \in \operatorname{Range}(A)$, so that there exists $v$ with $A v=g$. Uniqueness of $v$ follows from $A$ having full column rank.

Since $g^{k} \in \operatorname{Range}\left(A^{k}\right)$, there exists a $v^{k}$ such that $A^{k} v^{k}=g^{k}$. Since $A^{k}$ must have full column rank for all $k$ sufficiently large, the uniqueness of $v^{k}$ follows. Moreover, there is a constant $\beta$ such that for all $k$ sufficiently large, we have

$$
A^{k} s=t \Rightarrow\|s\| \leq \beta\|t\| \text {. }
$$

Since

$$
g^{k}-g=A^{k} v^{k}-A v=A^{k}\left(v^{k}-v\right)+\left(A^{k}-A\right) v,
$$


so that

$$
A^{k}\left(v^{k}-v\right)=g^{k}-g-\left(A^{k}-A\right) v
$$

Hence, we have

$$
\left\|v^{k}-v\right\| \leq \beta\left(\left\|g^{k}-g\right\|+\left\|A^{k}-A\right\|\|v\|\right),
$$

which implies that $\left\{v^{k}\right\} \rightarrow v$, as claimed.

\section{References}

1. M. AnITESCU, On using the elastic mode in nonlinear programming approaches to mathematical programs with complementarity constraints, Preprint ANL/MCS-P864-1200, Mathematics and Computer Science Division, Argonne National Laboratory, Argonne, IL, USA, December 2000. Revised December, 2003 and November, 2004. To appear in SIAM Journal on Optimization.

2. - Global convergence of an elastic mode approach for a class of mathematical programs with complementarity constraints, Preprint ANL/MCS-P1143-0404, Mathematics and Computer Science Division, Argonne National Laboratory, Argonne, IL, USA, April 2004. To appear in SIAM Journal on Optimization.

3. A. DeMiguel, M. Friedlander, F. J. Nogales, and S. Scholtes, An interior-point method for MPECs based on strictly feasible relaxations, Preprint ANL/MCS-P1150-0404, Mathematics and Computer Science Division, Argonne National Laboratory, Argonne, IL, USA, Argonne, IL, USA, April 2004.

4. R. Fletcher AND S. LeYfFer, Nonlinear programming without a penalty function, Mathematical Programming, Series A, 91 (2002), pp. 239-269.

5. — Numerical experience with solving MPECs as NLPs, Report NA/210, University of Dundee, August 2002.

6. M. Funushima AND J.-S. PANG, Convergence of a smoothing continuation method for mathematical programs with complementarity constraints, in Ill-posed Variational Problems and Regularization Techniques, M. Théra and R. Tichatschke, eds., vol. 477 of Lecture Notes in Economics and Mathematical Systems, Springer-Verlag, 1999, pp. 99-110.

7. M. Funushima And P. Tseng, An implementable active-set algorithm for computing a $B$-stationary point of a mathematical program with linear complementarity constraints, SIAM Journal on Optimization, 12 (2002), pp. 724-739.

8. D. M. GAY, Hooking your solver to AMPL, technical report, Bell Laboratories, Murray Hill, NJ, 1993. Revised 1994, 1997.

9. X. M. HU AND D. RALPH, Convergence of a penalty method for mathematical programming with complementarity constraints, Journal of Optimization Theory and Applications, 123 (2004), pp. 365-390.

10. H. T. Jongen, P. Jonker, And F. Twilt, Critical sets in parametric optimization, Mathematical Programming, 34 (1986), pp. 333-353.

11. - One-parameter families of optimization problems: Equality constraints, Journal of Optimization Theory and Applications, 48 (1986), pp. 141-161.

12. M. Kocvara AND J. OUTRATA, Optimization problems with equilibrium constraints and their numerical solution, Mathematical Programming, 101 (2004), pp. 119-149.

13. S. LEYFFER, MacMPEC AMPL collection of mathematical programs with equilibrium constraints.

14. G. H. Lin AND M. Fukushima, New relaxation method for mathematical programs with complementarity constraints, Journal of Optimization Theory and Applications, 118 (2003), pp. 81-116.

15. X. LiU AND J. Sun, Generalized stationary points and an interior-point method for mathematical programs with equilibrium constraints, Mathematical Programming, 101 (2004), pp. 231-261.

16. Z.-Q. LuO, J.-S. PANG, AND D. RALPH, Piecewise sequential quadratic programming for mathematical programs with nonlinear complementarity constraints, in Multilevel Optimization: Algorithms, Complexity and Applications, A. Migdalas, P. Pardalos, and P. Värbrand, eds., Kluwer Academic Publishers, 1998, pp. 209-229. 
17. J. Outrata, Optimality conditions for a class of mathematical programs with equilibrium constraints, Mathematics of Operations Research, 24 (1999), pp. 627-644.

18. J. Outrata, M. Kocvara, and J. Zowe, Nonsmooth Approach to Optimization Problems with Equilibrium Constraints: Theory, Applications, and Numerical Results, Kluwer Academic, Dordrecht, 1998.

19. M. L. Overton, Numerical Computing with IEEE Floating-Point Arithmetic, SIAM, 2001.

20. D. RAlPh AND S. J. WRIGht, Some properties of regularization and penalization schemes for MPECs, Optimization Methods and Software, 19 (2004), pp. 527-556.

21. S. M. RoBinson, Generalized equations and their solutions. Part II: Applications to nonlinear programming, Mathematical Programming Study, 19 (1982), pp. 200-221.

22. H. Scheel AND S. SCholtes, Mathematical programs with complementarity constraints: Stationarity, optimality, and sensitivity, Mathematics of Operations Research, 25 (2000), pp. $1-22$.

23. S. SCHOLTES, Convergence properties of a regularization scheme for mathematical programs with complementarity constraints, SIAM Journal on Optimization, 11 (2001), pp. $918-936$.

24. S. SCHOLteS AND M. STÖHR, How stringent is the linear independence assumption for mathematical programs with complementarity constraints?, Mathematics of Operations Research, 26 (2001), pp. 851-863. 